\title{
A Guided Tour of Selected Image Processing and Analysis Methods for Fluorescence and Electron Microscopy
}

\author{
Charles Kervrann, Carlos Óscar Sánchez Sorzano, Scott T. Acton, Fellow, IEEE, \\ Jean-Christophe Olivo-Marin, and Michael Unser, Fellow, IEEE
}

\begin{abstract}
Microscopy imaging, including fluorescence microscopy and electron microscopy, has taken a prominent role in life science research and medicine due to its ability to investigate the 3D interior of live cells and organisms. A long-term research in bio-imaging at the sub-cellular and cellular scales consists then in inferring the relationships between the dynamics of macromolecules and their functions. In this area, image processing and analysis methods are now essential to understand the dynamic organization of groups of interacting molecules inside molecular machineries and to address issues in fundamental biology driven by advances in molecular biology, optics and technology. In this paper, we present recent advances in fluorescence and electron microscopy and we focus on dedicated image processing and analysis methods required to quantify phenotypes for a limited number but typical studies in cell imaging.
\end{abstract}

Index Terms-Fluorescence microscopy, electron microscopy, image processing, image analysis, denoising, detection, segmentation, motion analysis, intra-cellular traffic analysis, cell motility analysis, neuron shape analysis.

\section{INTRODUCTION}

$\mathbf{T}$ HE use of microscopy imaging in biology is currently undergoing a revolution with the incorporation of all new kinds of microscopic techniques that allow the visualization, in vitro and in vivo, of tissues, cells, proteins and macromolecular structures at all levels of resolution (light, $\mathrm{X}$-ray, and electron microscopy), different functional states

Manuscript received May 07, 2015; revised September 14, 2015; accepted October 27, 2015. Date of publication December 04, 2015; date of current version January 21,2016. This work was supported in part by the Agence Nationale de la Recherche (French National Research Infrastructure France-BioImaging under Grants ANR-10-INBS-04 and ANR-10-LABEX-62-IBEID. The guest editor coordinating the review of this manuscript and approving it for publication was Prof. Fernando Pereira.

C. Kervrann is with the Serpico Project-Team Inria, Centre de Rennes-Bretagne Atlantique, 35042 Rennes, France (e-mail: charles.kervrann@inria.fr).

C. O. S. Sorzano is with the National Center of Biotechnology (CSIC), 28049 Madrid, Spain (e-mail: coss@enb.csic.es).

S. T. Acton is with the Department of Electrical and Computer Engineering, University of Virginia, Charlottesville, VA 22901 USA (e-mail: acton@virginia. edu).

J.-C. Olivo-Marin is with the Quantitative Image Analysis Unit, Institut Pasteur, 75724 Paris, France (e-mail: jcolivo@pasteur.fr).

M. Unser is with the Biomedical Imaging Group, École polytechnique fédérale de Lausanne (EPFL), CH-1015 Lausanne, Switzerland (e-mail: michael.unser@epfl.ch).

Color versions of one or more of the figures in this paper are available online at http://ieeexplore.ieee.org.

Digital Object Identifier 10.1109/JSTSP.2015.2505402

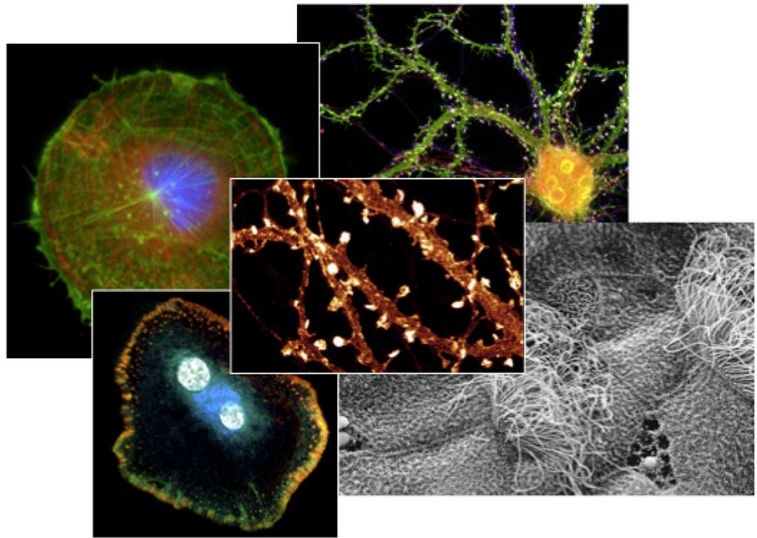

Fig. 1. An illustration of microscopy modalities used for investigation in cell biology at the sub-cellular and cellular scales (by courtesy of france-bioimaging. org).

through fluorescent labeling (GFP-Green Fluorescence Protein probes), chemical composition (fluorescence and spectral microscopy) and dynamic analysis (time-resolved microscopy). Furthermore, molecular (RNA interference, defective mutants), mechanical (micro-patterning) and optical (photoactivation) perturbations combined with multiparametric microscopy image acquisition allow one to confirm the established biological models and to discern whether the changes in expression have a role in the mechanisms under study. A more quantitative and integrated description, in space and time, of molecular interactions and coordination within macromolecular complexes at different scales observed in multidimensional microscopy, appears now essential for the global understanding of live mechanisms. The results will have a strong potential for applications in biotechnology and precision medicine: disease diagnosis, viral infection or defense mechanisms, detection of genomic instabilities, pathogenesis of hereditary genetic disorders, deterioration of cell cycle, epigenetic mechanisms, cancer prevention, neurodegenerative diseases and neurological disorder mechanisms...

To tackle all these stimulating challenges driven by technological progress in optics and molecular biology, constant efforts are necessary to develop innovative approaches and cutting-edge methods in multidimensional image processing and image analysis. In what follows, the term "image processing" is meant to convey low level operations on images (e.g., filters) which are often used for pre-processing type operations such as enhancements, denoising, deblurring, while the term "image 
analysis" is used for the remaining tasks which are related to information extraction, segmentation, tracking. ...

\section{A. Main Challenges in Image Processing and Analysis}

Advances in cell imaging have created specific challenges for researchers in image processing and image analysis. Indeed, light microscopy (LM) and electron microscopy (EM) have limitations that need to be overcome.

First, in image reconstruction the limitations of LM are driven by photon characteristics, such as spectroscopic properties of fluorescence, by the optical aberrations and the resolution of the overall microscopy system, and last but not least, by the photon budget available in the specimen. A direct consequence of cutting-edge acquisition implies the development of new concepts and algorithms for addressing challenging inverse problems including image denoising/deconvolution to preserve the integrity of samples in fluorescence and electron microscopy, accurate localization of proteins/molecules and motion analysis of single molecules. In EM some prior knowledge needs to be more used during the reconstruction process like the fact that macromolecules are constituted by atoms, the statistical distribution of coefficients in real space, Fourier space, etc. as well as their local structure in a sort of a priori distribution of local neighborhoods. Traditional image processing techniques are the core of methods in LM and EM, but they still need to be improved and adapted significantly to face the revolution of modern microscopy imaging.

Second, LM and EM are characterized by the nature of the observable objects (cells, organelles, single molecules, ...) in 2D/3D+time, by the large number of small size and mobile elements (chromosomes, vesicles, ...), by the complexity of the dynamic processes involving many entities or groups of entities sometimes interacting, by particular phenomena of coalescence often linked to image resolution problems, finally by the association, dissociation, recomposition or constitution of those entities (such as membrane fusion and budding). One typical challenge in LM is to detect and track xFP tags with high precision in movies representing several gigabytes of image data. However, low signal-to-noise ratios (SNR) and multiple objects confound tracking and it is hard to associate measurements with the correct objects, i.e., solve the data association problem [1]. More generally, post acquisition analysis is a limiting factor in front of the complexity of LM and EM data. In most cases, semi-automated image analysis workflows (e.g., "image denoising — object detection—object tracking—-trajectory classification") with minimal human intervention are designed to facilitate the interpretation of the information associated to large image corpus. On-line processing methods, cutting-edge algorithms as well as speed-ups of the currently existing ones are frequently developed to generate morpholog$\mathrm{ical} /$ dynamical features that need to be matched to proteomics/ trasncriptomics/genomics information.

\section{B. Positioning and Paper Organization}

In recent years, image analysis in microscopy has gained importance and the number of image processing and image analysis papers submitted to dedicated journals [2] in this area but also to more generalist magazines, workshops and conferences including in microscopy and bioinformatics, increased signifi- cantly in the last decade. The IEEE International Symposium on Biomedical Imaging (ISBI) focuses typically on microscopy image processing and analysis methods and cutting-edge algorithms. Moreover, several workshops/challenges are regularly organized. For instance, the DIADEM ("Digital Reconstruction of Axonal and Dendritic Morphology") challenge was first launched in 2009 to create algorithmic methods for automated neuronal tracing. The next initiatives "Particle Tracking" (ISBI 2012 [1]), “Cell Tracking” (ISBI 2013, 2014, 2015 [3]), “3D Deconvolution Microscopy" (ISBI 2013, 2014), "Single Molecule Localization Microscopy" (ISBI 2013), "3D segmentation of Neurites in Electron Microscopy" (ISBI 2013), are particularly useful to define the state-of-the-art algorithms to be included into analysis workflows, to compare standard and recent cutting-edge algorithms and to specify future progress and advances for specific topics. Moreover, related books [4], [5] and special issues [6]-[9] include a few tutorial-style overview articles covering progress in recent years for a large variety of topics (e.g., tracking in fluorescence bioimaging [10]-[12], sub-diffraction limited imaging and single molecule localization [13], [14], parametric active contour-based image segmentation [15]...). Finally, several authors presented independently state of the art methods for specific and important topics including cell-shape analysis [16], neuron tracing [17], co-localization (percentage of co-detection of interacting protein types at the same location) [18], [19], 3D image deconvolution [20], spot detection [21] in fluorescence microscopy... Even if it is generally a difficult task to present a broad view of activities in bio-image processing and analysis [2], several authors [22]-[25] already explained successfully how computer vision, image analysis and visualization algorithms combined in workflows, will play a significant role in image-based studies of cell biology.

In this paper, our ambition is not to cover all the topics in bio-imaging but to present with more details a few important image processing problems and applications not addressed in the aforementioned challenges and surveys (e.g., [18], [21], [1], [3]). Accordingly, we focus arbitrarily on dedicated image processing and image analysis methods that generally are included into workflows developed for specific biological studies. The remainder of the paper is organized as follows. In Section II, we present previous works, recent advances and challenges related to instrumentation in electronic and photonic microscopy. They include computational aspects on the reconstruction in LM, EM and correlative approaches. In Section III, we focus on dedicated image processing methods and algorithms required to quantify phenotypes observed at the intracellular and cellular scales. Four typical biological systems and applications are considered: cell integrity preservation during acquisition, molecular mobility quantification, cell motility analysis, analysis of neuron functionalities. In Section IV, we give the motivations for developing methods for computational biology and bioimage informatics. Section V contains concluding remarks and perspectives.

\section{AdVANCES IN EleCtRon AND FluORESCENCE MicROSCOPY AND INSTRUMENTS}

The last two decades have especially witnessed the explosion of the digital microscopy imaging: ion milling, multi-photon, 

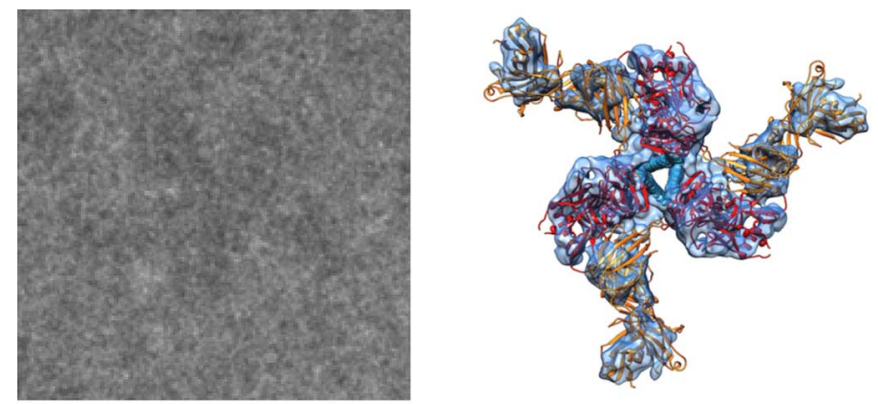

Fig. 2. Left: Example of a projection of HIV-1 envelope glycoprotein as seen at the electron Microscope. Right: corresponding 3D reconstruction at $6 \AA$ A resolution from several thousands of such projections [41].

stimulated emission depletion (STED) microscopy, structured illumination microscopy (SIM), 3D Multi-angle Total internal reflection fluorescence (TIRF), photo-activated localization microscopy (PALM) and stochastic optical reconstruction microscopy (STORM), near-field scanning optical microscopy, transmission full-field X-ray microscopy (TXM), scanning transmission X-ray microscopy (STXM), scanning photoelectron microscopy, micro-X-ray fluorescence, synchrotron radiation X-ray tomographic microscopy (SRXTM), etc. Current technological advances include structured and more coherent beam sources, faster and more sensitive detectors, the use of smaller and more sensitive molecular probes, automation of image acquisition. Additionally, the same device may include multiple different kinds of microscopes providing complementary information about the sample being visualized. In this section, we present trends in EM and LM image reconstruction.

\section{A. Image Reconstruction in Electron Microscopy (EM)}

Electron microscopy of frozen hydrated samples has proved to be a very effective tool in Structural Biology to elucidate the three-dimensional structure of biological macromolecules. Structural information is key to fully understand the physiological function of these macromolecules, as well as their pathological misfunctions. The electron microscope produces 2D images with very low contrast and very low SNR (see Fig. 2 left) on which image processing and tomographic algorithms are applied in order to produce a high-resolution 3D volume compatible with the measurements acquired by the microscope (Fig. 2 right). The image processing workflow includes automatic identification of high-resolution micrographs, the identification of the electron microscope transfer function, identification of particles in the micrographs, evaluation of their quality, 2D classification and identification of projection outliers or contaminants, construction of a low-resolution initial model, refinement of this model and classification of particles into different conformational states [26], [27]. These steps are normally performed using integrative packages (like Spider [28], Eman [29], Imagic [30], Xmipp [31]-[33], Bsoft [34], Sparx [35], Frealign [36] or Relion [37]) where several options for each step are available. There are projects like Appion [38] or Scipion [39] where all these packages are given a unified user interface.
The current resolution limit is about $3 \AA$ (the radius of an atom is between 1 and $2 \AA$ ). However, in the last 3-4 years, the whole field has experienced a technological revolution due to the introduction of Direct Detector Devices (DDDs) and the number of structures solved in the 4-3 $\AA$ range has rapidly grown in the last 2 years [40]. This technological advance has been accompanied by more accurate and robust image processing algorithms that help to better exploit the information contained in the micrographs. We may group the image processing advances in the last 3 years into those aiming at improving the resolution and those aiming at improving the throughput and robustness. Altogether, the goal is to have a robust high-throughput, high-resolution electron microscopy. The following sections summarize the advances in the last 3 years in the two aspects.

\section{1) High-Resolution Electron Imaging:}

Direct Detection Devices (DDD): As already mentioned, the recent introduction of Direct Detection Devices allows directly imaging electrons without the need of an scintillator that transforms electrons into photons with a significant blurring of the image. A number of works have characterized the performance of these new cameras [42]-[44]. Interestingly, due to the small exposure times of these new devices, it has been realized that the specimen actually moves inside the ice matrix [45] due to its interaction with the electron beam (most likely, this has been one of the most resolution limiting factors in years). Algorithms, as well as experimental ways [46], aimed at correcting this drift have been explored [47], [48], [43], [44], [49], [50] and it is still an active field of research.

Image Formation Process: As the data quality improves, very accurate image formation models have been proposed as a way to increase the resolution achievable. These models explore how electrons interact with matter (the macromolecule and its hydration layer [51]) and finally produce an image that is recorded by the device [52], [53]. For the moment, none of the previous advances in image formation has made its way into a $3 \mathrm{D}$ reconstruction algorithm, but certainly having a high-resolution model of how the image is formed in the microscope is a necessary step forward. Experimental procedures to reduce radiation damage have also been put forward as a way of better preserving the structural information contained in images [54].

\section{2) Robust High-Throughput Imaging:}

Image Acquisition: Acquisition in current electron microscopes is fully automated in the sense that several grids with frozen samples can be loaded at once, and a robot takes one by one these grids, put them inside the microscope, dedicated software will analyze the grid, decide the interesting regions and acquire electron micrographs. The precision of this process, keeping the samples at liquid nitrogen temperature and stabilizing the microscope operation over an extended period of time (up to $36 \mathrm{~h}$ without human intervention) has been a technological challenge (still ongoing) but to a certain extent achieved. These long microscopy sessions produce thousands of micrographs that have to be analyzed. The quality of the acquired micrographs and some sort of automatic selection of micrographs need to be performed [27]. Current methods primarily rely on features calculated on the Power Spectral Density of the micrographs. Then, the defocus of each micrograph is automatically estimated [55]. From each micrograph, a number (ranging from a few tens to a thousand) of individual particles are identified 
(like the one in Fig. 2 left). In total between 20k and 1M particles are extracted from the set of micrographs (normally using some automatic or semiautomatic algorithm [56] based on pattern matching, normally using rotational invariant features). In case that micrographs are taken as tilt pairs (one micrograph at zero tilt and the next one at some non-zero tilt angle), automatic methods have been put forward for identifying particle correspondences [57]. State-of-the-art algorithms produce a False Positive rate of about $15 \%$. Algorithms aiming at identifying those incorrectly identified particles have been investigated in [58], [59].

2D Image Analysis and Clustering: Particles extracted from micrographs are normally analyzed in 2D (the most common operation is a clustering) in order to have a summary of the data collected and as an exploratory tool to realize the possible different conformations and possible contaminants. This step is relatively settled in the field. Notwithstanding, some new developments have been introduced in the recent years addressing the speed of the clustering process [60], which is an important issue as the number of particles grows over $1 \mathrm{M}$, the stability of the clustering [61] and the presence of outliers [62]. Additionally, some works have explored the possibility to denoise particles as a way to improve SNR [63].

Estimation of the Initial Volume: A key step in the image processing of single particles is the starting volume. The refinement process is iterative and the choice of the starting volume can drastically bias the results towards a totally incorrect reconstruction corresponding to a local minimum of the landscape of solutions. Recent years have been very active in the development of robust algorithms for constructing a suitable initial volume without any a priori information [64]-[71]. They all exploit some sort of stochastic optimization and/or the special geometrical constraints imposed by the Central Slice Theorem.

$3 D$ Reconstruction: As the number of particles grows, new $3 \mathrm{D}$ reconstruction methods are developed in order to make the process more efficient [72], [50]. Another research line is making the reconstruction process more robust to noise by promoting sparsity in the reconstructed volume [73]. In order to remove unreliable particles, those whose angular assignment is not well understood may be removed from the process [74], this increases the robustness of the 3D reconstruction step.

Analysis of $3 D$ Heterogeneity: Macromolecular structures are flexible objects (flexibility allows physiological functions) and EM is a specially well suited technique to capture many different conformations. However, this advantage has to be matched with image processing techniques capable of identifying the different conformations (otherwise, they would all contribute to a single, blurred volume). This is currently one of the main open problems in the field and many new algorithms are trying to tackle the problem. Major trends come from i) a discretization of the conformational space through Maximum Likelihood or Maximum A Posteriori framework [75], [76], ii) the analysis of the continuous conformational space [77], [78], iii) or the analysis of the covariance matrix of the reconstructed volume [79]-[81].

Validation and Quality Assessment: Three-dimensional reconstruction in Single Particle Analysis is a technique that may easily fall into local minima. A major concern in the field is validating the correctness of the reconstruction obtained. In this re- gard, there has been a number of works aiming at providing criteria to check the validity of the reconstructed volume [82]-[85]. Once, we are confident about the result, we may wonder which is its resolution. The concept of local resolution has recently made its way into the field [86].

Analysis of Volumes in Structural Biology: Finally, we may try to recognize secondary structure elements [87]-[89], we may identify components by constraining the identification with other experimental sources like mass spectroscopy [90] and proteomics and chemical cross-linking [91], we may add $a$ priori knowledge about atomic models that should fit into the 3D reconstruction [92]-[96], or analyze its possible deformation paths [97], [98].

3) Subcellular Imaging and Electron Tomography: Electron microscopy is also used to image thin cellular slices. This technique is called Electron Tomography and it takes projections from the same slice at different tilt angles (typically between $-60^{\circ}$ and $\left.60^{\circ}\right)$. In the recent years, the main algorithmic effort in Electron Tomography has concentrated on a technique called subtomogram averaging. A tomogram is the $3 \mathrm{D}$ reconstruction of the cellular slice imaged at the microscope. In these slices we may see multiple copies of the same biological entity (for instance, ribosomes) in different conformations (each entity is in a different state of its biological cycle). We may extract from the tomogram each one of these entities, called subvolumes. These subvolumes have relatively low SNR, although larger than the SNRs in the micrographs, and an important lack of information in Fourier space (called the missing wedge). Subvolumes in the same conformation can be averaged as a way to improve the SNR, the resolution and to fill the missing regions in Fourier space. This problem is known as Subtomogram Averaging and may involve subvolume alignment (taking care of not comparing the missing areas) as well as subvolume classification. In the last years several subvolume alignment and/or classification methods have appeared [98]-[101]. Special attention has been paid to identify model bias [102] and to understand the limitations of subtomogram averaging [103]. We may think of subtomogram averaging as an alternative to Single Particle Analysis. Subnanometer 3D reconstructions using subtomogram averaging have been obtained [104]. However, the res-

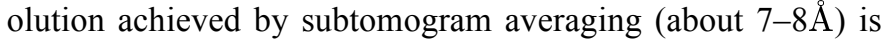
still far from the current resolution achieved by single particle

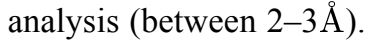

A different technique whose first pioneering works have recently appeared is called Single Molecule Tomography [105]. This technique is similar to Electron Tomography in its image acquisition scheme, but it differs in that, unlike Electron Tomography, a single molecule, not a cellular slice, is imaged. In this way, 3D reconstructions are obtained for each individual molecule. Then, these reconstructions can be combined using the same algorithmic techniques used for subtomogram averaging. At this moment, this technique is not widely used probably for several experimental (need for image acquisition automation, limitations in dose and molecular weight) as well as algorithmic (difficulties to locate and align the individual images due to the low contrast and SNR caused by the dose fractionation) constraints. 
Finally, some 3D reconstruction techniques have been published to deal with the discrete tomography problems that steams from energy filtered acquisitions [106], [107], some other algorithms try to get rid of reconstruction artifacts due to the missing wedge [108] and the use of gold beads to align the images [109].

4) Discussion and Perspectives: In the long run, the ideal for EM of single particles would be a situation in which several grids of the sample are loaded into the microscope and, automatically, this collects images that are processed online to produce one or multiple 3D reconstructions analyzing the dynamics of the structure at high-resolution. This process, ideally, would be mostly unattended and the image processing algorithms and microscope collaborate to produce high resolution structures without contaminants and sorting the different heterogeneous populations into different classes, even providing continuous paths along which the structure fluctuates. These high resolution volumes could serve as the basis for structure-based drug design by the pharmaceutical industry.

In the short run, different building blocks will pave the way towards this long run vision. At the level of microscope, phase plates [110] will help to work with smaller molecules yielding better contrasted images. These images along with their current standard images counterparts will allow regular reconstruction to 4-5 $\AA$, as long as there is no heterogeneity in the sample. Sample heterogeneity and sorting of the different conformational states as well as continuous changes will remain an open issue for long time, and new image processing algorithms are expected in this area. DDD cameras are expected to improve and give even better resolution data. In order to break the $3 \AA$ resolution barrier, we will need to locally estimate second and higher order microscope aberrations and incorporate into the $3 \mathrm{D}$ reconstruction algorithm the nonlinearities of a more physically based image formation model. 3D Reconstruction algorithms should exploit basic properties of standard molecules (like being sparse in some appropriate basis) and regularize the $3 \mathrm{D}$ reconstruction so as to get rid of as much noise as possible. The analysis of the different structure conformations is currently one of the most resolution limiting factors and new algorithms will appear to handle the molecular heterogeneity. Altogether, technological improvements at the microscope level along with image processing improvements of the data produced as well as the better integration of the EM results with already existing biochemical information will make EM one of the techniques of choice when developing new drugs using structural information of the target.

\section{B. Recent Trends in Sub-Diffraction and Correlative Microscopy}

The advances in optics, digital sensing technologies and labeling probes (XFP-Colored Fluorescence Protein) [111] (Nobel prize in chemistry 2008) have enabled to provide a very fine description of the components of the cell at the scale of a few nanometers to several hundreds of nanometers for a variety of applications in cell biology (see Fig. 1). In this section, we present recent trends in fluorescence, sub-diffraction and correlative microscopy.
1) Diffraction-Limited Microscopy and Previous Works: Investigating the impact of molecule clustering using diffraction-limited microscopy on the signaling pathway and beyond, was a tedious challenge, and even impossible in some cases. The most advanced available techniques, which allow for measuring molecular protein properties in the living cell, could offer information on concentration (FCS-Fluorescence Correlation Spectroscopy), dynamics (FCS, FRAP (Fluorescence Recovery After Photobleaching)), interactions (FCCS-Fluorescence Cross-Correlation Spectroscopy) and protein proximities (FRET-Fluorescence Resonance Energy Transfer) (see [112] for an overview). However, those correlation-based techniques have their drawbacks: i) some of them are invasive (FRAP-related techniques); ii) the data they produce are poorly compatible with precise spatial localization (FRET. . .); iii) most of them have strong protein concentration constraints (FCS, FCCS); and iv) some are based on rather slow measurement that are incompatible with molecular dynamics (photon counting-based FLIM (Fluorescence Lifetime Imaging Microscopy)) and related physiological consequence studies, at the whole single cell level. Characterizing the mobility and spatial organization of the implicated molecular actors, was not easily supported. Therefore, improving the optical resolution beyond $200 \mathrm{~nm}$ diffraction limit, while retaining the advantages of LM and the specificity of molecular imaging, has been a long-standing goal.

2) Sub-Diffraction Fluorescence Microscopy: Sub-diffraction limited microscopic techniques fulfill some of the requirements such as to close the resolution gap between conventional LM and EM methods. SIM (structured illumination microscopy) [118], [119] was first able to produce multi-color 3D imaging reconstruction of fluorescently labelled specimens with a lateral resolution approaching $100 \mathrm{~nm}$. Other advances-STED [113] (photo-switchable probes), STORM [114], [115] (inorganic photo-switchable dyes) or PALM [116], [117] (photo-activable fluorescent probes, Nobel prize in chemistry in 2014) - allow localization of single-molecules inside the cells at the scale of several dozens of nanometers. Therefore, they give access to precise determination of the spatial organization of the actors of signaling pathways on the membrane, at the single-molecule level. Whereas d(irect)-STORM produces static images, PALM can also be used to reconstruct single-molecule trajectories on the membrane ("single-particle tracking"(spt)-PALM). In general, single fluorescent molecule are detected in each image frame and tracked using a dedicated algorithm [235] and Gaussian fitting. The localization accuracy, depending on the SNR, is of the order of $20-25 \mathrm{~nm}$, that is an image resolution of $40-50 \mathrm{~nm}$. Trajectory are then computed using nearest neighborhood methods and consists of successive positions of 5-100 points in an average. The great advantage of sub-diffraction microscopy is to potentially be compatible with chemical treatments that putatively alter clustering and trafficking and can be used to measure the effect of this treatment at the level of the whole cell response, thus ensuring experimentally that the chemical treatment indeed had the expected effect on clustering.

The first limitation of sub-diffraction microscopy is that it naturally put severe constraints on tagging. For instance, 
u-PAINT ((universal Point Accumulation for Imaging in Nanoscale Topography) [124] is able to reach slighter resolution than spt-PALM and provides dynamical information on a single live cell with large statistics revealing localization-specific diffusion properties of membrane biomolecules; nevertheless, u-PAINT needs to be coupled to antibodies, which can generate biological artifacts. d-STORM is appropriate to map endogenous proteins using fused fluorescence probes, smaller than antibodies, which can be reversibly photo-switched. Albeit used on fixed (dead) cells, d-STORM gives very fine spatial localization accuracy $(20-30 \mathrm{~nm})$ with two to three colors. Finally PALM is not suited when one wants to use two or three different colors/markers, which can be quite useful co-localization/clustering studies. Finally, a typical single cell spt-PALM experiment leads to a set of several thousands of images that need to be processed in order to extract molecule localization and dynamics.

Actually, all these point-by-point approaches (PALM, STORM) aim at improving the resolution to the detriment of acquisition rate. For example a $28 \times 28$ micrometer square image with a lateral resolution of $60 \mathrm{~nm}$ is recorded in approximately 1 minute with PALM technique [120]. Even SIM is poorly compatible with time regimes expected in most live cell imaging, which restrict its application mostly to fixed samples. Advances in information restoration will probably make sub-diffraction imaging more compatible with the imaging of molecular dynamic in live cells in the future. Very recently, high-NA (numerical aperture) TIRF-SIM [121], PA (pattern activation) non-linear SIM citeLiBetzig2015 and lattice light-sheet microscopy (LLSM) [122] permitted to image respectively with high spatial and temporal resolution the dynamic associations of cortical filaments with proteins (high-NA TIRF-SIM, $97 \mathrm{~nm}$ resolution), the remodeling of the actin cytoskeleton (PA NL SIM, $62 \mathrm{~nm}$ resolution) and the intracellular dynamics in 3D (LLSM). In this line of work, an innovative way to perform high-resolution in photonic microscopy, while keeping access to the accurate dynamic ranges within single cells, is based on azimuthal spinning TIRF imaging [123]. Unsurpassed TIRF image quality is provided by fast rotational laser illuminations, minimizing (by averaging) the fringe aberrations commonly observed in TIRF microscopy. A dedicated 3D reconstruction algorithms have been proposed and enable to visualize and quantify fast cellular events localized at or close to the plasma membrane of live adherent cells (up to $1 \mu \mathrm{m}$ in depth with at least $30 \mathrm{~nm}$ axial resolution) [125]. The temporal resolution of the high resolution 3D TIRF (up to 35-40 frames per second) makes this technique appropriate for studying the coordination of vesicle recycling at the plasma membrane and cytoskeleton dynamics, at the same time and in depth. also compatible with dynamics study at the single cell level and the very low photobleaching and phototoxicity.

3) Full Correlative Microscopy and Hybrid Strategies: To define the temporal frame of a biological event, the spatial information at both the nano-or micrometric scales needs to be correlated with optical microscopy of living cells that will be immobilized in a time resolved way. To extract biophysical information of one particular molecular species in its macromolecular context and thus to understand complex biological systems, there is a necessity of multiparametric measurements that capture diffusion, flow or exchange of proteins, protein-protein interactions in time and space, in living cells. The information gathered at these different levels is not coherent and cannot presently be integrated easily. A key approach is to correlate information extracted from diverse microscopy techniques, beyond advanced LM.

Combination of Diffraction-Limited Microscopes: While a lot of image acquisition technologies giving access to various scales of biological material exist, pinpoint and manipulation of the biological samples, through the different setup is only achieved through tedious and timeless experimental procedures. Consequently, multiparametric instruments enabling biologists to use different techniques simultaneously or sequentially as fast as possible, and to render them applicable to high throughput analyses, are currently investigated. Ideal instruments would be automatic positioning "multimodal microscopes" based, for example, on multipoint confocal and Total Internal Reflection Fluorescence (TIRF) microscopy, using diverse laser-assisted techniques (4 Dimensional, TIRF-Dual Channel, TIRF-FLIM, FRAP, Photo-conversion and activation, optical tweezers). Combined approaches including FLIM, FRET and live LM have started to demonstrate that macromolecular complexes determine an ultrastructural architecture whose function gives rise to the integrative scale of the life matter within cell and tissues.

CLEM Microscopy: Another very active field in the domain of microscopy imaging has been to search for ways of combining EM with LM (CLEM) [126] to combine the advantages of live fluorescence microscopy with the high resolving power of EM [126]. Extensive research has been performed in designing probes that can be seen in both modalities, designing sample holders that can be used in both microscopes, the physical construction of microscopes with the two kinds of imaging, etc. A major limitation within CLEM strategies is the time interval between cell selection under the light microscope and the fixation step (chemical fixation but also transfer to the high pressure freezing apparatus) that takes a few seconds and up to minutes, far too slow to fix rapid intracellular movements at the exact time of interest. To make it short, more integrated instrumentation and protocols for sample preparation and handling have been proposed. In this context, a topological averaging of cell structures and standardization of cell shape, allowing rapid and easy re-localization when passing from one scale to the other, has been made available through micro-patterning technologies [127].

\section{Perspectives for Image Processing and Analysis Methods}

To better elucidate the role of specific proteins inside their multiprotein complexes and to decipher the dynamic coordination and organization of molecular complexes at the cell level, image processing and image analysis methods, mathematical models, and algorithms are increasingly investigated to build an integrated imaging approach that bridges the resolution gaps between the molecule and the whole cell, in space and time [23]. Facing the amount of information provided by high-throughput multidimensional microscopy, the methodological approach is to link in a single workflow, information extracted from 
imaging molecules by sub-diffraction limited resolution LM using cutting-edge detection algorithms, eventually at a time regime compatible with live cell imaging.

The overview of ultrastructural organization is achieved by complementary electron microscopical methods. Since there is a large resolution gap between conventional LM compatible with live cell imaging and EM, microscopists needs fast, robust and efficient computational methods and algorithms to locate and pinpoint structures of interest, to improve image contrast, to remove noise for a better interpretation of the image contents and to register images [128], [129]. At first glance, information theory criteria [130] seem more appropriate to analyze images coming from different modalities. A number of comparison studies in medical imaging (CT/MR imaging or US/MRI imaging) shown that the similarity measures yield different performances depending on the considered modality combinations [131]. Furthermore, data fusion and LM-EM image matching are challenging issues and correspond to a large variety of scales, image contents and noises. As shown in [132], LM images are typically blurred when compared to EM images.

\section{Focus on A Few Image Processing AND ANALysis METHODS AND APPLICATIONS IN BIOLOGICAL IMAGING}

Image processing and analysis applied to microscopy has become a key tool in molecular biology since it enables to quantify biological processes in space and time at the subcellular and cellular levels. Theories, methods and algorithms have been developed to face multidimensional spatial-temporal data provided by imaging sensors and technologies as described below. Nevertheless, microscopists and biologists are at present flooded with data that they have to normalize, filter, denoise, deblur, reconstruct, register, segment, classify, etc. All these operations are currently performed by basic and/or advanced signal and image processing algorithms, which are gaining more and more importance as the collected data becomes more sophisticated and more complex in its acquisition and image formation model. These operations are generally gathered into image analysis workflows for specific biological experiments.

In this section, we present a few very popular bio-image processing methods generally integrated into workflows to analyse the structure and dynamics of molecules, proteins and cells. We focus on four biological studies:

- Preservation of cell integrity (photo-toxicity versus exposure time) using image denoising methods;

- Traffic analysis from particle motion and molecule trajectories in live-cell imaging;

- Cell motility analysis using tracking algorithms;

- Analysis of structure and functionalities of neurons using segmentation algorithms

They represent typical biological studies which are conducted in cell imaging and cell biology. For each study, we give biological issues, methodology and perspectives.

\section{A. Preservation of Cell Integrity in Live Cell Imaging}

1) Motivation: Many live-cell fluorescence imaging experiments are limited in time to prevent phototoxicity and photobleaching. It is established that the amount of light and time required to observe entire cell divisions or intracellular mechanisms or processes can generate biological artifacts [133]. It has been demonstrated that image denoising allows images to be taken more frequently or over a longer period of time, while preserving image quality [134]-[136]. Such post-acquisition processing can improve the frame rate by a factor of 100 times [133]. Conversely, for a given acquisition rate, it can permit to reduce the sensitivity threshold, allowing imaging for long time regimes without photodamages. This strategy has been successfully applied to wide-field and Nipkow disk based confocal [137], regular laser scanning confocal microscopy [138], TIRF (Total Internal Reflection Fluorescence microscopy [139], and 3D-PALM microscopy [140]. The major advantage of denoising algorithms is to potentially acquire images at very low SNR while recovering denoised $2 \mathrm{D}+\mathrm{T}(\mathrm{ime})$ and $3 \mathrm{D}+\mathrm{T}$ (ime) images. Such developments will be also required to be compatible with "high-throughput microscopy" since several hundreds of cells are observed at the same time and the exposure times are typically reduced.

2) Image Denoising Methods in Fluorescence Microscopy: In this section, we present the state-of-the-art methods adapted to remove Poisson and Poisson-Gaussian noise generally measured in fluorescence microscopy. Image denoising is generally the first step of many image analysis workflows since it helps to visualize the phenomena and mechanisms under study.

Noise Modeling: An imaging set-up consists generally of an optical system followed by a photodetector and associated electrical filters. The photodetector converts the incident optical intensity (i.e., photons) into electrons. Unfortunately, the signals are generally damaged by many different sources of noise during acquisition. Poisson noise typically arises in adverse conditions such as poorly illuminated environments, short exposure times, and low-efficiency photon detectors. This is especially true in microscopy. In LM imaging, signals are known to be corrupted by intensity dependent Poisson noise but also by additional sources of electron noise [141]. Formally, the most popular model in fluorescence microscopy is as follows

$$
z(\mathbf{x})=g_{0} \aleph(\mathbf{x})+\epsilon(\mathbf{x})
$$

where $z(\mathbf{x})$ is the intensity observed at space-time location $\mathbf{x} \subset \mathbb{R}^{d}, g_{0}$ is gain of the overall electronic system, $\aleph(\mathbf{x})$ is the number of photo-electrons at pixel $\mathbf{x}$ assumed to be Poisson distributed with unknown mean $\theta(\mathbf{x}), \epsilon(\mathbf{x}) \sim \mathcal{N}\left(m_{\epsilon}, \sigma_{\epsilon}^{2}\right)$ is a white Gaussian noise and represents "dark current". Such a noise is signal dependent and requires to adapt the conventional denoising approaches.

Variance Stabilization Transform: In the literature, most of methods have been developed for Poisson noise reduction. The key challenge in Poisson intensity estimation problems is that the variances of the observed counts are different. The effect of Poisson noise increases (i.e., the SNR decreases) as the mean intensity value decreases. The first category of methods dealing with Poisson noise are based on variance stabilization techniques [142], [143], [137], [144], Anscombe transform [145] and Haar-Fisz transformation [146]. The idea consists in converting Poisson noise into a Gaussian noise with unit variance [142], [147], [143], [137], [144]:

$$
f(\mathbf{x})=2 \sqrt{z(\mathbf{x})+3 / 8} \sim \mathcal{N}(0,1)
$$


and then applied commonly-used denoising algorithms for processing additive for additive white Gaussian noise. Stabilization techniques provides satisfying results when the number of collected photons is high enough [137], [144]. Furthermore, it is also assumed that the data are corrupted by additive white Gaussian noise with signal dependent variance such as: $z(\mathbf{x})=$ $u(\mathbf{x})+\epsilon(\mathbf{x})$ and $\epsilon(\mathbf{x}) \sim \mathcal{N}(0, u(\mathbf{x}))$. Many conventional denoising methods for white Gaussian noise are then adapted for this situation of high count numbers (e.g., see [78]). For very limited-photon imaging, this approximation does not hold and alternative approaches are required for restoration. In the most severe cases, the measurements are 0 is most locations in the image or 1 corresponding the arrival of one photon. In fluorescence imaging, this situation is not usual but is considered in Time-Correlated Single Photon Counting (TCSPC) - Fluorescence Lifetime Imaging Microscopy (FLIM) [148], [149]. A alternate approach is to consider Poisson noise statistics (or Poisson-Gaussian noise statistics [150]-[152]) and maximum likelihood estimators [153] or Maximum a Posteriori estimators [154]. The idea is to directly handle Poisson noise without "Gaussianization" of the data, which is more appropriate for low SNRs.

Patch-Based and Wavelet-Based Methods: To date, the most competitive methods for dealing with Poisson noise fall in the two following categories:

- Patch-based methods: In the case of additive white Gaussian noise, the NL-means filter [155] is considered as an efficient and simple approach for noise reduction while preserving image geometry. This method exploits image redundancy captured by patches to restore information. In order to optimally perform in the case of Poisson noise, the NL-means has been combined with Fisz transform [156], [157]. Other authors proposed to combine more efficiently Principal Component Analysis [158] and dictionaries [159] to patch-based representation to reduce Poisson noise. In [160], the authors proposed an extension of the NL-means based on probabilistic similarities to compare patches. Additional adaptation of NL-means for two-photon microscopy [161] and FLIM imaging has been proposed in [162], [163].

- Wavelet-based methods: Traditionally, wavelet-based methods are recommended for image denoising [164] including for Poisson noise reduction [165], [143]. The combination of a risk estimate for Poisson statistics with the Haar multiresolution provided fast (PURE) algorithms with applications in fluorescence microscopy [166], [167]. In this area, [168] and [169] addressed the problem of mixed Poisson-Gaussian noise.

In another line of work, compressive sensing is currently investigated to save time and measurements in biological imaging [170], [171].

The case of 2D-3D multiframe analysis has been addressed with patch-based [137] and wavelet-based [172] and was especially dedicated to fluorescence imaging (see Fig. 3). Nevertheless, additional efforts are required to adapt the above mentioned mathematical frameworks mostly designed for 2D images corrupted by Poisson or Poisson-Gaussian noise to be compatible with time-lapse cell imaging and "high-throughput microscopy".

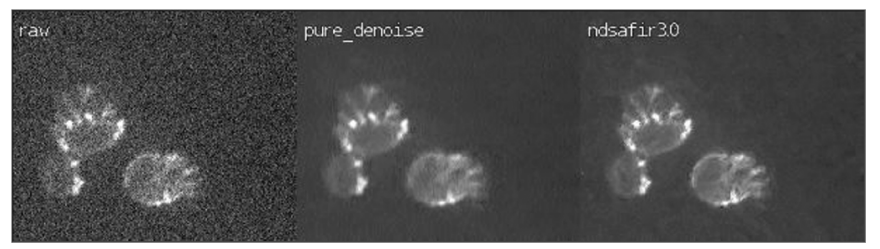

Fig. 3. Comparisons of two multiframe denoising methods. Left: original image; middle: wavelet-based method [172]; right: patch-based method [137].

3) Denoising-Deconvolution Methods and Perspectives: To preserve live samples during image acquisition, the illumination intensity must be set to safe levels. This induces increased noise in images and a severe loss of resolution to the extent that the subcellular components appear to be blurred. Several methods [20], [173] and software [174], [175] have been proposed to improve signal to noise ratio and resolution assuming a Gaussian noise or a Poisson noise (e.g., Richardson-Lucy algorithm [176], [177]). In recent years, the most popular deconvolution methods in fluorescence microscopy are based on the minimization of an energy functional being the sum of two terms: A data fidelity term depending on the image formation process (e.g., noise statistics, point spread function) and a regularization term imposing some prior on the solution. Generally, a additional constraint is considered to promoting positivity of the solution. In this framework, several regularizers have been investigated to suppress large amounts of noise while restoring spatial details and structures: Tikhonov, wavelet-based [178], Total Variation (TV) (e.g., [179]), second-order derivatives (e.g., Laplacian, Hessian [180]) eventually combined with entropy-based potentials [181]. Mixed norms combining first and second order derivatives have been also designed to attenuate stair-case effects of the TV norm. Finally, optimization algorithms are required and need to be customized for image reconstruction (e.g., see [178], [181], [180], [179]).

Interestingly, the combination of patch-based denoising (sparse coding denoising) and deconvolution algorithms in [179] produced impressive results on the synthetic datasets of the "3D deconvolution microscopy" challenge (ISBI 2013). In [137], the deconvolution of denoised 3D+time images significantly improved the image resolution in real experiments. Building on these success, a new line of work will be to combine intelligently denoising and deconvolution algorithms in an appropriate signal processing framework to recover structural details and improve spatial resolution, while at the same time pushing the illumination to extreme low levels in order to limit photo-damages and photo-toxicity. Denoising-deconvolution methods can actually improve the quality of any type of microscope and new results on STED [113] and SIM [118] will demonstrate in the forthcoming years the novel synergy between sub-diffraction limited imaging techniques and image deconvolution as already investigated in [182]-[184], [125]. Finally, the high computational time will require the development of accelerated versions of the most used restoration algorithms in microscopy to face massive data (e.g., 4D wide-field microscopy) to process.

\section{B. Intracellular Traffic Analysis and Molecular Mobility}

In live cell imaging, the analysis of fluorescence fluctuations in time in a given volume allows measuring motion and den- 
sity of the fluorescently labeled molecules and proteins inside the sample. The methodological approach (e.g., image analysis workflow) is then to i) detect, ii) track and iii) classify dynamics of proteins and molecules using dedicated image processing methods, at a time regime compatible with live cell imaging. Quantitative analysis of molecule or groups of molecules trajectories is the next issue. In this section, we present an overview of methods for i) detecting spots/molecules and for iii) molecular mobility estimation. An complete overview of fluorescently labeled proteins tracking (item ii)) is given in [1].

1) Biological Issues: Eukaryotic cells are characterized by membrane bound organelles. Their abilities to divide and fulfil their various functions within integrated tissues rely on the tight regulation of membrane composition, on the generation of ubiquitous and specialized organelles and on their capability to communicate with each other. Current research efforts in cell biology have already contributed to identify hundreds of components defining key machineries of essential functions.

A new long-term goal in fundamental biology is to decipher the dynamic coordination and organization of interacting molecules within molecular complexes at the single cell level and to explore the role of transport intermediates (e.g., vesicles) to higher levels of complexity, as during remodeling of the plasma membrane, differentiation and cell migration in contexts in forced two dimensions (micro-patterns), or in reconstituted three-dimensional environments. Targeting of specific proteins is essential for cell homeostasis, specialized tissue function and development of living organisms. This is achieved by protein sorting through the different intracellular organelles of the secretory and endocytic pathways of non-polarized and polarized eukaryotic cells and vesicular transport of soluble and membrane components. It has emerged during the last 20 years that transport from one compartment to the next one follows similar mechanistic principles, that is formation of coated vesicles, which bud from a donor compartment and then fuse with the recipient compartment. They involve similar protein networks controlling soluble and membrane protein sorting and vesicle formation, transport vesicle movement along cytoskeleton elements (actin nucleation machineries and molecular motors) and membrane fusion. Nevertheless, it is still difficult and challenging to understand how these different machineries using multiple protein-protein and protein-lipid interactions are interconnected and coordinated in time and space during a given reaction like for intracellular transport, for instance. Also are unclear the mechanisms by which these processes are regulated in highly differentiated cells in order to properly function in a tissue.

2) Spot and Particle Detection in Microscopy Images: Investigations in LM at the single cell level have been faced with the problem of estimating the location and dynamics of spots, such as microtubule end-tips, adhesion molecular complexes, or vesicles. Intra-cellular objects of interest inside the cells are generally small and often appear as bright spots, which can be round or elongated, with intensity that varies along time over a possible time-varying and cluttered background.

Detecting subcellular particles in fluorescence microscopy is a crucial task for further quantitative analysis including particle counting [185], particle pattern recognition [186], particle tracking [187], [188], [1] or dynamics classification [189]-[192]. For instance, in [193], a point process modeling is proposed in order to characterize some properties of the endo/exocytosis process such as the presence of clusters from detecting spots. Generally, the manual detection of objects over a cluttered time-varying background is very tedious especially in $3 \mathrm{D}$ and subjective. Automatic methods have the obvious advantage of being quicker and reproducible. Several approaches have been then developed recently for detecting multiple small moving subcellular objects. Specific applications include for cell segmentation [194], [195] and nuclei detection [196]-[198]. Comparisons of spot detection methods have been reported in [21], [199]. We give a complementary overview of methods in this area, from basic methods (e.g., image thresholding) to more established methods (e.g., LoG filtering, wavelet- and morphological-based methods).

Image Histogram Analysis: In fluorescence microscopy, existing methods usually assume that the background is static and the moving object intensity is brighter than the background. The central question is then how to adjust the threshold to extract the desired objects. The simplest way of detecting spots is to threshold the image intensities from the histogram. The threshold value can be automatically found by using the Otsu's method [200], entropy minimization [201], [202] or Expectation Maximization (EM) algorithms [203], [204]. Unfortunately, thresholding is not a good approach when the SNR is low. Actually bright pixels do not necessarily belong to spots, and pixels in spots can be very dim.

In most real images the background is not uniform and a single global threshold fails to detect the objects of interest. Therefore, numerous space-varying thresholding methods were studied [205], [206]. If local thresholding methods demonstrate a higher performance than global thresholding methods when the background is spatially varying, cluttered backgrounds composed of structures of different sizes are still challenging and generally lead to poor results. To avoid misclassification of isolated pixels, spatial coherence between neighboring pixels must be taken into account. A common approach consists in first applying a low-pass filter to the image, such as a Gaussian filter. However, the cutoff frequency parameter is critical for accurate localization and detection.

LoG Filtering and Local Maxima Detection: More advanced methods not only smooth the image, but also enhance the underlying signal. In [207], Thomann et al. defined the objects of interest as the local maxima of intensity where the local curvature (defined as the determinant of Hessian matrix) is high. Instead of local curvature, the Laplacian of Gaussian (LoG) [189], [208] has shown a good ability to determine the object locations. In this area, the LoG filter is a band-pass filter which enhances objects of a particular size, reduces noise and lowers low-frequency background structures. Sage et al. [189] empirically observed that the LoG filter is close to the optimal whitened matched filter for Gaussian spots in fluorescence microscopy images. Yet, the choice of the LoG filter bandwidth is critical and highly dependent on the spot size. In [209] the authors have then proposed a locally adapted threshold automatically inferred from local intensity statistics and a given probability of false alarm (see Fig. 4). Recently, a Markov Point Process that uses multiscale blobness images obtained by LoG filtering has been described to reduce the number of missed detections [210]. 

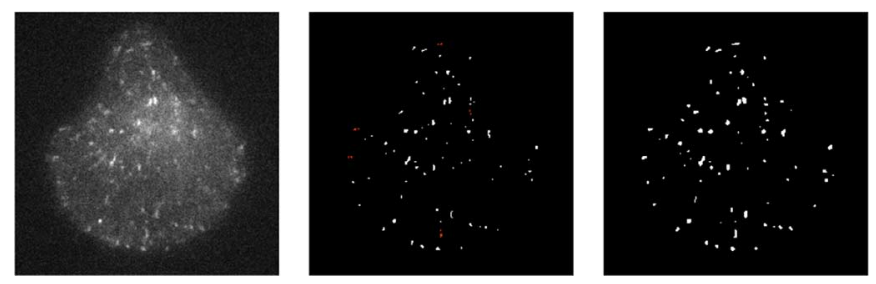

Fig. 4. Comparison of [147] (MSVST (middle)) and [209] (LoG-based method, right) (2D-TIRF image depicting vesicles in a M10 cell, left).

Isotropic Undecimated Wavelet Transform: Wavelet transform is also a very attractive and powerful approach and a simple tool to decompose the image into different scales and orientations. In bio-imaging, wavelet decomposition has proved to be particularly appropriate for object detection [211], [212], [147], [213]. In [211], [147], an isotropic undecimated wavelet transform (IUWT) [214] is mainly exploited to detect objects of various sizes. A wavelet multiscale product operation in [211] is performed at each location to reveal correlations across the scales. For a given wavelet scale, spots respond more significantly to IUWT than uncorrelated noise. However, noise can have a higher response than spots at smallest scales in very noisy images inducing undesirable detections. It follows that the smallest scales must be discarded to decrease the false detection rate. Finally, the wavelet multiscale product map is thresholded to get a binary detection map. In [147], the authors described the so-called MSVST method which relies on variance stabilization techniques to rule out insignificant coefficients of IUWT (see Fig. 4).

Furthermore, the image is reconstructed by discarding the coarsest scales corresponding to the background structures and the smallest scales corresponding to noise. The spots are finally detected by thresholding the reconstructed image. It worth noting that with both IUWT-based methods [211], [147], the set of wavelet scales must be chosen carefully with respect to the spot size.

Mathematical Morphology and h-Dome Operators: In another line of work, methods that directly detect objects and estimate background are based on mathematical morphology [215], [216]. In biological imaging, the "Top Hat" method [217], [218] is especially well recommended to extract objects in 2D images by applying an erosion operator and a dilation operator with a disk-based structuring element. By considering the image as a 3D structure, the image intensity being the third dimension, the "Rolling Ball" method [219] performs a morphological open transformation using a ball-based structuring element. This is probably the most popular method to subtract image background in fluorescence microscopy.

A more sophisticated and powerful method [21] is based on the so-called " $h$-dome" operation [216], [21]. In [21], a Gaussian blur is first applied to reduce noise. The image background is then estimated by an opening operation which removes objects smaller than the structuring element. The image background is finally subtracted to detect spots. Peaks with an amplitude higher than a given value $h$ are selected. The " $h$-dome" map contains domes with small areas corresponding to noise, domes corresponding to spots, and domes with large areas corresponding to complex and irrelevant structures in the background. To remove the undesirable domes, the authors proposed to generate samples from the " $h$-dome" map viewed as an importance sampling function. In this approach [21], the algorithm parameters must be carefully chosen to provide excellent results. Note that the objects of interest do not correspond to a unique value of $h$, so that the method sometimes merges very bright neighboring spots, and sometimes misses dark spots. To overcome this problem, [220] have proposed an adaptive method for selecting the most appropriate value $h$. Furthermore, the spatial image gradient amplitude is thresholded, which provided more satisfying results in the case of abrupt changes in the background.

Spatio-Temporal Detection: The signal in time-lapse microscopy typically varies with time due to photo-bleaching and molecular processes or dynamics. So the threshold must be adapted to each frame according to the current image intensity and contents. A normalization procedure can be also applied in some cases to handle photobleaching and global intensity variations. In this area, extension of spot detection methods to space-time analysis has been investigated in time-lapse fluorescence microscopy.

Generally motion detection by background subtraction is well addressed in video-surveillance [221]-[223] and was recently investigated in Poisson video [224]. Nevertheless, mainly due to the background intensity changes over time, the extension of existing methods to fluorescence image sequences needs specific adaptation. Typically, no occlusion occurs as in natural scene video images and one generally assumes that the signal emitted by the background and the fluorescent objects are additive signals at each pixel in the image. Background and spots are then jointly estimated using parametric models [225] or non-parametric approaches (e.g., "rolling ball" [219]). In video analysis, Markov random field modeling and global energy minimization methods are usually recommended to capture local statistics and to represent spatial correlations in video analysis [222], [221]; this framework has been studied in fluorescence microscopy in [226].

More specifically, some methodologies can be considered for the automatic detection of appearing and vanishing spots of fluorescently labelled clustered molecules in wide-field (WF) and TIRF microscopy images [193], [190]. The difficulty is to distinguish motions due to trafficking from the appearing and vanishing spots. Two-frame approaches and frame difference thresholding methods [227] are then more appropriate to detect fast appearing and vanishing spots time-lapse microscopy. More dedicated methods taking photobleaching and photon-limited nature of images into account via a variance stabilization transform has been successfully proposed in [190]. Complementary approaches in this line of work are currently investigated to detect "packing" and "un-packing" molecular events [228].

Discussion and Limitations: All the referenced methods have in common critical parameters whose optimal value is closely related to object sizes. If the object scale is chosen too small, over-detection occurs induced by noise. If this value is too high, objects are smoothed out or merged when close to each other. In practical imaging, the scale parameter can often be inferred from image data or provided by the biologist-expert. Note that spot detection methods produce binary detection map after thresholding the wavelet-based, LoG or morphological filter responses. 
In biological imaging and spot detection, the datasets used in the experiments remain limited yet in terms of content and challenges [21], [199], [1]. Indeed, real images are far more complex than images of this dataset, specifically, the SNR is generally lower in real images and objects to be detected are smaller and often darker. Additional more realistic and more challenging datasets with ground truth and simulators to quantitatively evaluate and compare methods are under study.

Application to Co-Localization and Perspectives in SubDiffraction Imaging: In fluorescence imaging, co-localization defined as the percentage of co-detection of interacting protein types at the same location, quantifies usually the presence of two molecules in very close proximity [229], [18]. Co-localization occurs when two or more molecules bind to the same structure or domain, without interacting, but somehow being spatially correlated.

In the literature two categories of co-localization approaches are generally considered, which are either intensity-based or segmentation-based [18], [19]. The occurrence of yellow signals in an overlay fluorescence image generally depicts the correlation between the locations of the green and red signals in the cell, thus showing some co-localization between the two proteins under study. Correlation measurements (Manders' descriptors [229]) are conventionally used in fluorescence microscopy. In order to quantify the above phenomenon, object-based methods have been applied in the literature (see [230] and [231], [19], where the spots detected as described above, are reduced to points and their interaction is analyzed by spatial statistics methods (point processes, descriptive Ripley's functions [232]). In this area, False Discovery Rate framework to test a set of distances between pairs of molecular entities could be more investigated [233].

At nano-scales, co-localization is a novel and challenging problem that needs to be more correctly addressed with the emergence of sub-diffraction limited microscopy methods. Super-resolution microscopy that rely on specific probes, like PALM [116] (photo-activable fluorescent probes), naturally put severe constraints on tagging, and cannot be easily combined with commonly-used co-localization techniques. In general, the notion of co-localization needs to be redefined when dealing with dual-color super-resolved and computationally reconstructed images at the scale of 10-20 nm. Nevertheless, an important issue is how to take into account the size of antibodies [234] and the segmentation (e.g., "watershed" algorithm [235]) errors in the analysis.

3) Estimation of Molecular Mobility: For communication between cell compartments, the transport intermediates corresponding to small spherical or turbo-vesicles, propelled by molecular motors (e.g., kinesin, dynein, myosin...), move along microtubules or microfilaments. As vesicles are transported away on microtubules and have been observed to co-localize near fusion sites, it is most probable that the cytoskeleton may in part function to specify a domain-specific fusion site. Finally, while being also stochastically defined, the traffic is known to be oriented and it is established that local dynamics of intermediate transports obey to biophysical laws, including confined and free diffusion.

Measuring diffusion (or Brownian motion) and transport (or directed flow) is central in cell biology (e.g., axonal transport of motor proteins) because since they represent the main modes of mobility of molecules in living cells. In this section, we present the problem of estimating diffusion coefficient and directed flow often representative of local change of the medium in time-lapse fluorescence microscopy. Four categories of methods are generally considered. We present briefly each approach and discuss their advantages and limitations.

Single Particle Tracking (SPT): is based on the identification of the position of single particles, fluorescently tagged, and the analysis of their trajectory over time. Given computed tracks by nearest neighborhood algorithms or more sophisticated and more robust to noise methods [1], the mean-square displacement (MSD) of tracks is generally used to appropriately interpret and detect free and confined diffusion and directed flow [236]. For a given trajectory $X(\Delta t)$ composed of $N$ points with a temporal sampling $\Delta t$, MSD is defined as:

$$
M S D(k \Delta t)=\frac{1}{N-k} \sum_{i=1}^{N-k}\|\mathbf{x}(k \Delta t)-\mathbf{x}((i+k) \Delta t)\|_{2}^{2}
$$

where $\|\cdot\|_{2}^{2}$ is the square of the Euclidean distance.

Inside the cell, diffusive dynamics (Brownian motion) is a key component in short distance transportation (e.g., connectivity for signal transduction). The diffusion phenomenon has been described by Robert Brown in the early 19th after the stochastic motion has been observed for pollen particles. It has been later demonstrated that this motion is due to the thermal agitation in the medium resulting in shock between molecules and causing stochastic trajectories. By integration of the squared displacement along the trajectory over time, we get the fundamental Stokes-Einstein equation:

$$
M S D(t)=2 n D t
$$

where $n=2$ in $2 \mathrm{D}$ and the scalar diffusion coefficient $D$ is linked to the viscosity of the medium and the size of the particle. Unrestricted free diffusion is then indicated by the linearity of the plot of MSD. In confined environment (i.e., disk of radius $R$ ), it has been demonstrated that MSD will saturate [237]: $M S D(\infty)=R^{2}$. When the density of trajectories is high (e.g., spt-PALM [234]), two-dimensional maps of the molecule mobilities can be produced by displaying in a color-coded pixel the local mean instantaneous diffusion constant of the molecules detected in that pixel. These types of maps can provide for example dynamic information of subcellular regions of a given cell, in order to see if there is correlation between mobility and location [234].

Another dynamical process that have been heavily studied in the past decade is the motor-mediated transport (e.g., dynein, kinesin, myosin) of molecules. Primarily supported by actin filament and microtubule network, it ensures spatial organization and temporal synchronization in the intracellular mechanisms and structures. The observed displacement presents a locally constant speed $v$ along the cytoskeleton and we have [238]: $M S D(t)=v^{2} t^{2}$. Nevertheless, the complexity of internal structures and molecular processes in the living cell influence the molecular dynamics and prevent the systematic application of pure Brownian or directed motion modeling. In the Brownian diffusion case, intracellular clutter can cause anomalous diffusion resulting in MSD measurements that differs from 
the theory. On the one hand, cytoskeleton density will hinder the free displacement of the particle resulting in a non-linear evolution of the MSD below the theoretical expectation, a phenomena called subdiffusion. On the other hand, the cytoskeleton elasticity combined with thermal bending can contribute to active diffusion which will result in higher MSD measurements than predicted by a normal diffusion process [239]. To discriminate dynamics, it is usual to fit MSD curves with the more general model [240]:

$$
M S D(t)=t^{\alpha}+\beta
$$

where the constant $\alpha$ determines the most probable motion model: confined diffusion $(\alpha<0.1)$, obstructed motion $(0.1 \leq \alpha \leq 0.9)$, Brownian motion $(0.9 \leq \alpha \leq 1.1)$, directed motion $(\alpha>1.1)$. Generally, it is recommended to filter out the last points of trajectories but eight points are required at least for fitting. Finally, statistical methods for robust fitting and correlations modeling are presented in [241]. Another frequent intracellular scenario is the jerky motion of molecules switching between diffusion and motor-mediated motion [238].

Spatio-Temporal Image Correlation Spectroscopy (STICS): The most popular methods for diffusion analysis are based on correlation measurements under the hypothesis of temporal stationarity of fluorescence signals [242], [243]. The so-called Spatio-Temporal Image Correlation Spectroscopy (STICS), derived from fluorescence correlation techniques, is widely used in fluorescence imaging to recover physical parameters such as directional flow or diffusion parameters of moving molecules. This method does not require any particle or object tracking and integrates the variations of fluorescence over space and/or time via correlation measures to access to information at the molecular level, such as diffusion coefficients or dominant flow speed and direction [242]. The generalized spatial and temporal correlation expression is defined as

$$
r(\mathbf{w}, \tau)=\frac{1}{N-\tau} \sum_{t=1}^{N-\tau} \frac{\langle\delta I(\mathbf{x}, t) \delta I(\mathbf{x}+\mathbf{w}, t+\tau)\rangle}{\langle I(\mathbf{x}, t)\rangle\langle I(\mathbf{x}, t+\tau)\rangle}
$$

where $I: \Omega \times[1, N] \rightarrow \mathbb{R}$ is an image sequence of $N$ frames with $\Omega$ the image domain, $\mathbf{w} \in \mathbb{R}^{2}$ are the spatial lags, $\tau$ $\in[1, N]$ is the temporal lag, $\langle\cdot\rangle$ is the spatial average over a patch and $\delta I(x, y)=I(x, y, t)-\langle I(x, y, t)\rangle$. We point out that $r(\mathbf{w}, \tau)$ is not a normalized correlation criterion but enables to recover the biophysical parameters associated to density, motion of molecules, and diffusion coefficient [242].

For transport estimation, the goal is to estimate the translation vector corresponding to the correlation peak maximum. The static or immobile molecule population is usually filtered by local averaging and $r(\mathbf{w}, \tau)$ is computed by Fast Fourier Transform. A 2D Gaussian function is considered to estimate accurately the correlation peak over time [242] using a Levendberg-Marquardt optimization scheme. In the experiments, the analysis is performed on image blocks and the size of the blocks determines the scale of moving objects retrieved (see Fig. 5). The spatial lag between blocks is chosen to achieve an acceptable trade-off between spatial accuracy and computational time.

In a diffusive motion scenario, the following heat diffusion equation is satisfied: $I_{t}(\mathbf{x})=D \Delta I(\mathbf{x})$ where $I_{t}$ is the temporal

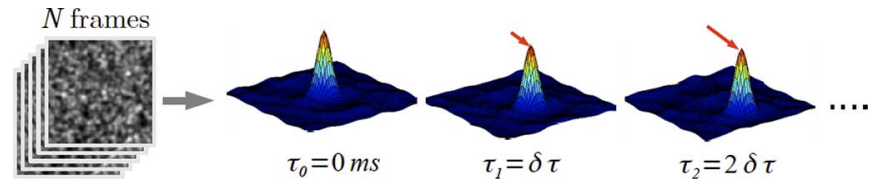

Fig. 5. Motion estimation with STICS. Tracking of the correlation peak by Gaussian fitting on correlation maps.

derivative of $I, D$ is the isotropic diffusion coefficient and $\Delta$. denotes the Laplacian operator. In order to estimate the scalar value $D$, the diffusion decay $\tau_{d}$ is derived from the following equation obtained by combination of (5) and the heat equation (see [244] for details):

$$
r(0,0, \tau)=r(0,0,0)\left(1+\tau / \tau_{d}\right)^{-1}+r_{\infty}(\tau)
$$

where $r_{\infty}(\tau)$ is the long-time offset and $D=\overline{w_{0}} / 4 \tau_{d}\left(\overline{w_{0}}\right.$ denotes the temporal average of $\omega_{0}$ (laser beam size)).

Fluorescence Recovery After Photobleaching (FRAP): consists in analyzing the fluorescence recovery after its photobleaching in an specified area by using a high intensity laser pulse. FRAP analysis is performed directly by measuring the mean intensity in the photobleached region over the image sequence. The extracted fluorescence recovery curves are normalized to 1 for the pre-bleached intensity. Furthermore, all recovery curves are then adjusted using a non-linear least square algorithm to the theoretical model proposed in [259].

Discussion and Comparison: The interest of STICS is mainly the estimation over temporally extended subsequences, which introduces a regularization effect and increases robustness to noise. On the counterpart, the number of frames $N$ must be carefully chosen such that motion can be approximated by a constant translation over the sequence. This assumption of temporal stationarity of motion is quite restrictive in practice. Contrary to STICS, optical flow produces dense diffusion fields and can adapted to transport or Brownian motion estimation. Spatial variations can be recovered accurately, in particular at discontinuities usually occurring across membranes. Unlike STICS, optical flow exploits only two frames, which is either an advantage but also a limitation since considering more frames would produce better results.

A comparison between FRAP, FCS (Fluorescence Correlation Spectroscopy), RICS (Raster Image Correlation Spectroscopy) and SPT was described in [260] for the estimation of translational mobility in 3D in neurons, but without considering any data. This paper was aiming to help the reader to choose a technique for practical use. SPT was recommended to detect different classes of diffusion without considering a parametric model unlike FCS or RICS methods; the analysis is based on the slope of MSD curves over time.

Perspectives for Image Processing and Analysis: A majority of approaches for motion analysis in biological sequences is based on individual tracking of biological objects (see e.g., [10], [246], [245]); the potential and limitations of particle tracking algorithms have be well described in [1]. However tracking methods are not always adapted for motion analysis, especially when the density and the lack of prominent features prevent the individual extraction of objects of interest undergoing complex motion. Accordingly, estimating motion fields can be then more appropriate to capture complex dynamics 
observed in biological sequences [247], [248]. The usual approach for optical flow estimates the dense motion field by minimizing a global energy functional composed of two terms:

$$
\widehat{\mathbf{w}}=\arg \min _{\mathbf{w}} E_{\text {data }}(I, \mathbf{w})+\lambda E_{\text {reg }}(\mathbf{w}) .
$$

where $\mathbf{w}: \Omega \rightarrow \mathbb{R}^{2}$ is the dense motion field, $E_{\text {data }}$ is a data term penalizing deviations from a data conservation assumption over time, $E_{r e g}$ is a regularization term enforcing smoothness of the flow field and $\lambda>0$ serves as regularization parameter to balance $E_{d a t a}$ and $E_{r e g}$ contributions. A high value of $\lambda$ allows to retrieve only dominant motions of large structures by smoothing the flow field, while a small value of $\lambda$ tolerates repeated close spatial variations corresponding to small objects. Applications of global regularized method in biological imaging have recently been investigated in [249], [250], [248], [251]-[254]. Because of possible intensity changes (e.g., photobleaching), the data term needs to be adapted [255]. The data term [256] based on the assumption of conservation of intensity and spatial gradient of the image is typically robust to additive illumination changes, which is necessary for several biological applications [257]. In a pure diffusive motion scenario, the global energy can be specialized. Rather than estimating a constant diffusion coefficient over patches as performed with the STICS method, the idea in [258] is to consider a dense diffusion field $D: \Omega \rightarrow \mathbb{R}$ instead of $\mathbf{w}$. Reconciling optical flow, SPT and STICS methods appears to be a promising and stimulating research direction in the field of motion analysis and classification. Also, SPT and motion analysis methods could be combined to neuronal tracing algorithms to accomplish higher-level objectives (e.g., see Section III.D).

\section{Cell Motility Analysis}

The study of the detailed mechanisms of cell motility has become a major research area in life sciences, as motion is an essential and critical feature of cellular processes (e.g., parasitic infection, immune response, or tumor formation) [261]. Over the past years, much attention has been focused on the molecular and physical mechanisms of cell motility. The mechanisms underlying cell movement are nonetheless poorly known, and are studied using a variety of experimental approaches, one of which being via the observation of living cells in their 3D environments using fluorescence microscopy.

1) Biological Context: A large amount of work has concentrated on the first step of movement consisting in cell polarization and its regulation based on the activities of actin-based subcortical cytoskeleton [262]. The dominant model of cell motility indicates that the forces driving membrane protrusion are generated by monomeric globular G-actin polymerizing onto filamentous F-actin at the cell front; closed to there, MyosinII pulls the rear of the actin network. In protrusive structures, multiple actin filaments are dynamically arranged in cross-linked webs (as in lamellipodia) or parallel bundles (as in filopodia). Among the several types of cell migration that have been identified, amoeboid motion [263] is characterized by a crawling-like displacement, and more precisely by the movement induced by the production of blebs, that are local bulges in the cell membrane [264], [265], [262]. These protrusions appear in a very fast and sudden manner, and are thought to be produced by the rupture of the links that attach the cell cytoskeleton to the membrane [266]. Besides the amoeboid motion, many cell types motility is based on the blebbing of the plasma membrane.

Dynamic analysis of membrane protrusion has recently shown that bleb expansions occur when the membrane detaches from the actin cortical cytoskeleton leading to bleb inflation [266]. As expansion ceases, contractile cortex reassembles under the membrane and drives bleb retraction. This is the case of amoeboid cells where membrane deformations at high frequency remind those from other cells where the plasma membrane detaches from the cytoskeleton, e.g., apoptotic cell [267], non-adherent carcinosarcoma cells [268], or melanoma cells showing prolonged blebbing while spreading [265]. These blebbing mechanisms are different of those described in [269], where no cortical breakdown prior to membrane bulging has been observed.

2) Cell Tracking Methods: In terms of image analysis, quantitative analyses of cell shape [16] and motion primarily require segmentation and tracking of individual cells. Typical 3D+time data sets consist of thousands or tens of thousands of images. However, and contrary to the sophistication of molecular biology and biophysics tools used by biologists, the image processing and analysis methods that are applied by end-users for motion analysis are rather simple. Commercial microscopy software packages generally feature tools for object segmentation and tracking, mostly based on standard image processing schemes that are performed manually or using semi-automatic procedures (see [282] for a representative list). As a result, cell tracking is still extremely labor-intensive, in addition to being prone to errors, user bias, and lack of reproducibility.

Besides methods based on thresholding and watershed, several methods mostly based on deformable models like snakes or level sets have been successfully demonstrated to outperform human analysis [270], [3]. The specific set of methods provided by active contours and deformable models is particularly adapted to the problem of tracking highly deformable cells. Deformable models, originating from the seminal paper [271], are closed fronts (curves in 2D or surfaces in 3D) that iteratively evolve in the image domain, usually according to a gradient descent algorithm designed to minimize an energy functional. This energy of the form:

$$
\hat{\mathcal{C}}=\arg \min _{\mathcal{C}} E_{\text {data }}(I, \mathcal{C})+\lambda E_{\text {reg }}(\mathcal{C}) .
$$

is composed of both image-related terms $E_{\text {data }}(I, \mathcal{C})$ where $I$ denotes the image, which tend to be minimal when the front $\mathcal{C}$ is at the object boundary, and image-independent terms $E_{r e g}(\mathcal{C})$ that embody prior information on the computed boundary and play the role of regularizers in ill-posed problems [272]. Deformable models provide a powerful and flexible framework, where suitable energy terms can be defined depending on the image characteristics and prior knowledge specific to a given application.

Two main categories of deformable models can be distinguished according to the mathematical representation of the contour or surface:

i/ explicit models where the boundary is represented by parametric functions (e.g., [271], [15]); 
ii/ implicit models where the boundary is defined as the zero level set of a scalar function defined over the image domain (e.g., [273]).

This framework has been adapted for cell tracking independently by several groups [270], [274]-[276].

Interestingly enough, the recent paper [3] reporting on a "Cell Tracking" challenge organized in conjunction with ISBI 2013, presents results where the standard techniques outperform more elaborated algorithms. This benchmark of cell tracking algorithms is based on the use of a common diverse video dataset repository and ground truth with specific criteria for the evaluation of the segmentation and tracking accuracy, and unified criteria for comparing and ranking the algorithms. Although this type of competition seems to indicate that computer-aided cell tracking has somehow become a mature field, in reality the impact of cell motility assessment has not been as high as expected, at least in terms of usefulness to biological studies. This is probably due to the fact that extracting positions, contours and speed is not enough to understand and model the mechanisms of cell motility.

A better understanding of this complex process calls for new approaches to extract and characterize the cell shape and the large variety of protrusions exhibited by cells. Indeed, it appears that cells and unicellular organisms usually exhibit an ordered and highly regulated cycle of complex shape changes in order to generate movement [261]. Hence, understanding cellular shape and movement has become an area of active research that requires efficient shape quantification tools to describe and classify the wide variety of shape configurations, with the aim of deciphering the biological mechanisms underlying cell motion.

3) Discussion and Perspectives: A number of pioneering studies in cell shape classification [278]-[281] based on the previous approaches have opened up the way to start and connect phenomenological features like the structure of blebs, the dynamics of the subcortical cytoskeleton and its links to membrane during protrusion to the underlying molecular mechanisms that determine and regulate them. They represent also a first step towards understanding how cells generate force for shape change and movement and how they respond to mechanical force stimuli. These topics certainly represent the next frontier for the community working at the interface between physics, biology, and image analysis.

In the context of highly deformable cells, such as cells exhibiting amoeboid motion, robust shape description and analysis is particularly challenging, due to the high degree of variability that can be observed within a so-called homogeneous population, while different populations may exhibit visually similar deformation patterns. To this end, shape description techniques have progressively shifted from contour-based shape representation and measurements to more advanced mathematical solutions based on frequency analysis such as spherical harmonics (SPHARM). The SPHARM transform considers any closed surface as a function of the unit sphere, and simplifies this function into a unique set of coefficients, facilitating subsequent shape characterization and classification. This technique offers interesting properties such as position and orientation invariance [266], and is thus well suited for shape sets with high variability such as living cells [263], [264]. They have been complemented by the spherical wavelets [277] that are constructed by analogy to wavelets in the plane via appropriate spherical projections, and are particularly well adapted to localize features along surfaces.

\section{Neuron Morphology and Structure Analysis}

Deciphering the functionalities of the brain has been an aspiration held by neuroscientists for many years. In the last decade, due to the significant progress in experimental biology and microscopic imaging techniques, this daunting challenge of mapping the brain (in simple organisms) appears more attainable. One may hypothesize that of the remaining technical challenges, several key developments must emerge from the signal processing community.

To solve the neuro-image problem in an automated fashion, several fundamental subproblems must be addressed to design a robust image analysis workflow: image acquisition, object segmentation and structural analysis [17]. Broadly, the relevant research in neuro-image analysis can be categorized into the following groups: segmentation and shape analysis of individual neurons, classification of neurons and characterization of the intra-neuronal structures.

1) Biological Context: The relationship between the morphology and functionality of neurons was postulated by Santiago Ramón y Cajal in the 19th century. Cajal's hypothesis serves as the basis for modern day neuro-image analysis. Morphological analysis of individual neurons and neuronal components such as dendritic spines, synapses, mitochondria among others has shown promise in better understanding and diagnosis of various neurological disorders and neurodegenerative diseases [283]-[288]. The scale of this problem varies between a few hundred neurons in the roundworm C. elegans to a hundred billion in an adult human brain. It is evident that neuro-image analysis becomes a big data problem as we transition into the study of vertebrates and eventually to humans. Such a scale in analysis is not approachable by manual laboratory observation.

2) Image Acquisition for Neuron Observation: Choice of a particular imaging modality depends on the specific application. Fluorescence microscopy is a popular choice when the study involves a global structural analysis of the neurons or some neuronal components in the micrometer scale. For such imaging techniques, the specimen is tagged with a fluorescence protein (GFP, YFP etc.) which emits photons when illuminated by a light source [289]. These photons are eventually detected by a sensor to produce an image of an optical plane. Laser scanning confocal microscopes are commonly used for fast three dimensional imaging of neurons of model animals such as Drosophila, rat, mice and others. Depending on the application, other imaging techniques such as bright-field microscopy [290], multiphoton microscopy [291] may also be used to image neuronal structures.

EM is a popular choice for imaging neuronal structures at nanometer scale. EM is particularly useful in analyzing subcellular objects and surrounding structures such as mitochondria, synapse, vesicles, etc. Focus Ion Beam Scanning Electron Microscopy (FIBSEM) [292] can now deliver near isotropic 3D images with extremely high resolution and is emerging as the imaging modality of choice for nano-scale analysis of neuronal structures. 
3) Segmentation and Morphology of Neurons: Global structural analysis of neurons requires a two-stage pipeline. First, a digital reconstruction should be obtained from the raw image data. This is the segmentation (often called tracing in the biological community) stage. With the reconstruction available, the next challenge is to devise a method to compare the structures. It turns out that both these subproblems are loaded with their own sets of challenges and complications

Neuron Segmentation: Neuron segmentation or neuron tracing refers to the problem of acquiring the neural geometry from raw image data. Recent emphasis is on analysis of 3D images and fluorescence microscopy is a commonly used imaging technique for such purpose. Image processing is challenging both due to the structural complexity of neurons as well as due to imaging artifacts such as poor contrast, presence of non-neuronal clutter and low signal to noise ratio of the images. The objective is to perform 3D segmentation of the neuron, which requires proper handling of the filament bifurcations as well as accommodating signal attenuation.

Most of the existing neuron tracers assume that neurons are tree-like structures. Semi-automated neuron tracers are popular with biologists since such techniques allow user intervention, thus providing more flexibility in tracing. Neuron tracing using semi-automated techniques generally requires the user to input some initial seed points. Subsequent seed points which reside on the neuron centerline are generated either automatically or with human aid [293], [17], [294]. With a set of points available to trace, graph based algorithms are then used with the seeds as nodes of the graph, to reconstruct the neuronal tree. Such semi-automatic algorithms [294]-[296] provide useful means for neural structure segmentation due to their speed and accuracy. However, it is argued that optimal seed point selection is difficult to automate and while human assisted methods can improve accuracy, such methods require significant technician labor.

A separate set of methodologies have also been proposed that focus upon neuron reconstruction without seed initialization. A preprocessing step is often deployed to eliminate noise and clutter, and the filament enhancing filter due to Frangi et al. [297] has been used extensively for neuron contrast enhancement and de-cluttering. The preprocessed image is used for segmenting the neuronal structures. Existing automated neuron segmentation methods use parametric and geometric active contours [298], [299], graph based methods [300], steerable filters [301], wavelets [302] etc. The general trend is to embed the centerline of the segmented neuron in a graph theoretic tree for further computational study. Open-source software suites such as Vaa3d and NeuronStudio have emerged recently to aid the neuroscientist in tracing.

Comparison of Structures: With the neuron structure segmented and its morphology defined by a tree, the next step is to design a platform to compare the morphology of the segmented neurons [303]. A comparison typically involves the computation of a similarity score for a particular type of neuron based on the examples from a training dataset. To build the training set, one needs to categorize the neurons on the basis of their functionality. This categorization is a challenging problem in itself, since neuron morphology varies significantly by organism [304], [305]. Recently, promising results have surfaced [306]-[310]. As more datasets of traced neurons are made available, this initial progress should blossom into robust comparison algorithms.

4) Detection and Classification of Dendritic Spines: Image analysis of dendritic spines is the backdrop for exciting recent developments in neurodegenerative disease studies. Neurons in the nervous system are arranged to form an interconnected network, with the axons and dendrites of the neurons connected via synapses. It is understood that synaptic activities are directly related to the morphological properties of dendritic spines, which are the small, elongated structures on the dendritic surface. Changes in spine morphology directly affect the synaptic activity, which, in turn, influences the global functionality of the neural system. These dendritic spines play an important role in studying different neurological maladies such as Alzheimer's disease and Parkinson's disease [311].

The spines are short, tubular protrusions from the dendritic filaments, which are visible in fluorescence microscopy. Therefore, many preprocessing tasks such as denoising and contrast enhancement for automated spine detection borrow preprocessing techniques for 3D microscopy imagery. Detecting dendritic spines can be a challenging problem since the structures are small in size and they are often difficult to distinguish from the background. Spine detection is often performed after detecting the dendritic branch, since spines are attached to the filaments. Automated detection techniques have been proposed which use morphological filters [311], region growing [312], [313], techniques based on spine geometry via key point identification [314], [315]. In many cases, it is necessary to deploy a post-processing step to ensure spine connectivity to the neuron shaft.

Once the spines are detected, the next step is to perform automated classification based on spine shape. A set of distinguishing features are generated based on the spine morphology (maximum curvature, convexity, area etc.) and the derived feature set for a particular spine is used as input for a classifier in order to predict the functional category [311], [316], [317].

5) Segmentation of Intra-Neuronal Structures: The third category of active image analysis research for neurons is the analysis of subcellular structures such as synapses, mitochondria, cell membranes, etc. The advances detailed to this point are aimed at global morphology. In contrast, with high resolution imaging by way of EM, it is possible to image neuronal structures at nanometer scale. This imaging can reveal rich information regarding neural network substructures like the synapses, mitochondria and vesicles. For example, changes in synapse appearance and distribution provide clues as to the neural development process. Shape and size based properties of mitochondria of neurons are indicators of the extent of neuronal degeneration.

A prominent issue in all EM analysis tasks is the sheer volume of the data, which necessitates powerful computer architecture and computationally efficient algorithms. The high resolution image stacks provide useful local information, since a number of subcellular structures are visible at the nanometer scale. Synapses are vascular structures that are located at the junction of two neurons. Synapse detection from 3D EM stacks have been investigated using interactive methods via active contours and recently, using statistical learning techniques that perform synapse detection using local contextual cues [318]-[321]. Other recent success stories include the detection 
of mitochondria and cellular membranes for the purpose of neural anomaly detection from EM [322]-[325].

6) Concluding Remarks and Perspectives: Several open problems exist in the analysis of neuron structure, connectivity and function. International research efforts are delivering online repositories and software challenge workshops ([326], [327], ISBI 2012, ISBI 2013, DIADEM challenge) as well as from the recently announced BigNeuron Project [328]. Propelled by advances in microscopy, signal processing will play a fundamental role in the reverse engineering of the brain.

\section{Image Processing and Analysis Methods vs COMPUTATIONAL BIOLOGY AND BIOIMAGE INFORMATICS}

In cell and molecular biology [23], new challenges arise to acquire a complete and quantified view from the scale of a "single" cell to the scale of a multi-cellular structure, within the whole organism. In the near future, image processing and analysis will be central to the successful use of LM and EM in post-genomics biology.

\section{A. Impact of Microscopy Image Processing in Systems Biology}

System biology is a field in expansion, which has evolved into various branches and paradigms to address problems at various scales ranging from ecology to molecular structures. It aims at modelling system as a whole in an integrative perspective instead of focusing on independent biophysical processes [329], [23]. In the future, new mathematical approaches and image processing and analysis methods are needed to deal with high degrees of complexity and uncertainty inherent to biological systems, especially for describing the interactions between the different components in the cell observed in LM and EM. An important goal is to bridge the gap between very detailed biophysical models and more integrative models. All the molecular dialogues under concern at different spatial scales (atomic level, protein level, compartments,) and temporal scales (from nanosecond to second) must be considered. One typical project is the cell in silico as investigated in Harvard Medical School (http:// vcp.med.harvard.edu/) or the VCell of the University of Connecticut Health Center (http://www.nrcam.uchc.edu/). In this line of work, several contributions to combine imaging, modelling, image processing (as presented in Section III), statistics and machine learning in cell biology need to be encouraged.

\section{B. Processing of Mass of Data in High-Throughput Microscopy}

In the emerging era of high-throughput microscopy (biochemical screens, cell-based screening [22]), systematic and accurate correlation and analysis of the data cannot be performed manually, since the image sequences are composed of several hundred of 3D stacks. Consequently, data to manipulate range from few to tens of terabytes. From the experimental perspective, molecular (drugs, RNA interference), mechanical (micro-patterning...), and optical (FRAP, photoactivation, optogenetic...) functional modulations allow one to quantify the importance of molecular linkage into macrocomplexes within a single cell. Even with high-speed computers, the intensive processing of very large images will considerably slow down the whole analysis process. Therefore, a special attention must be paid to the feasibility and scalability of the developed algorithms. Fast implementation on graphical units need to be investigated when necessary and the nowadays-widespread multicore processors must be exploited. The development of fast algorithms should enable the processing of image sequences in real time and offer new perspectives especially during the acquisition process.

Moreover, efficient storage, fast retrieval and secure sharing of microscopy images are also crucial challenges. An important challenge consists in developing a robust and hybridized architecture including an extensible, secure, and comprehensive data model with semantic and spatial queries on imaging data. In order to deal with the challenging problems mentioned above, strong efforts have been made to organize the micro- and macro-image into databanks (see OME-OMERO (http://www. openmicroscopy.org/site) [330], but none has yet proven fully satisfactory and functional. One important issue is then to define an image database with a built-in query system to annotate, retrieve, process and integrate analysis from different imaging modalities. The database will be able to search via meta-data and includes menu selections that enable to run remote processing from a cluster. Integrated image processing softwares in the interface environment allow the database users to process their images easily, and store associated results and parameters. The main tasks to address are:

- standardisation of image acquisition, annotation and storing protocols,

- development of a plan for interoperability of existing image analysis platforms and open platforms for biological image analysis,

- development of databases annotated with ground truth and gold-standard for validation and benchmarking of algorithms,

- development of access to infrastructures that support large scale image computing. The observation and understanding of the life matter at the nano- and the microscopic levels means a workflow of image data obtained along multidimensional microscopy modes.

The combination of complementary skills (image processing and analysis software, image data management) will yield a full integration of the image and data life-cycle, from image acquisition and analysis, to statistical analysis and mathematical modeling in systems biology.

\section{Software Issues}

Finally, connections with commonly-used free and commercial softwares will be made in order to make popular the algorithms [331]. This includes softwares for cell imaging (see OBIA http://www.openbioimage.org/):

- ImageJ / http://rsbweb.nih.gov/ij/

- Fiji / http://fiji.sc/wiki/index.php/Fiji

- ICY / http://icy.bioimageanalysis.org/

- MetaMorph (commercial)

- http://www.moleculardevices.com/Products/Software/Meta-Imaging-Series/MetaMorph.html 
- Amira (commercial) / http://www.amira.com/

- and softwares for electron tomography:

- Chimera / http://www.cgl.ucsf.edu/chimera/

- IMOD / http://bio3d.colorado.edu/imod/

- TomoJ / http://u759.curie.fr/fr/telechargements/softwares/ tomoj/tomoj-00733

- Digisens (commercial) / http:/www.digisens3d.com/en/ soft/2-Digi_ECT.html

- Several academic softwares will be also considered to adapt our dissemination strategy and extension for end-users:

- http://bigwww.epfl.ch/algorithms/

- http://www.bioimagexd.net/

- http://www.cell profiler.org/

- http://penglab.janelia.org/software/Hanchuan_Peng_ Software/software.html

The experimental set-ups need nowadays to be combined with adapted processing and analysis techniques for quantification and representation of image contents in a reproducible fashion. This includes object tracking, denoising and restoration, event detection, background estimation. .. Such a new generation of acquisition setups based on integrated solutions have a high potential for specific purposes of monitoring protein-protein interactions and molecular behaviour in cell biology. It can also serve for implementing high throughput methods for the identification of new biological targets and screening for chemical drugs able to interfere with such processes.

\section{General Conclusion and Perspectives}

New imaging techniques allow considering the precise structural organization of their functions and their progressive conversion as a function of temporally defined multi-molecular interactions. Such a complexity is however only understood if multiscale analysis and representation matches their molecular description, which requires an important effort in image processing and image analysis. The amount of information provided by medium or high-throughput multidimensional microscopy is constantly growing while offering new ways of investigating the cell mechanisms.

The originality of the field lies on the direct cooperation between cell biologists, applied mathematicians, image processing scientists and biophysicists, to understand the complexity of molecular machines involved in intracellular transport, from multiscale and multimodal microscopy. We will be concerned with the following topics:

- Developing new bioimaging approaches combining innovative optical and numerical methodologies, to observe the coordinated dynamics in live material.

- Correlating spatiotemporal organization of protein networks at micro scales with the architectures of their biological environment at the ultrastructural scale: this requires rapid and easy processes to manipulate from one scale to the other one, as well as to fuse and easily visualize data obtained at different scales and modalities, in space and time. New approaches to register LM images and EM images will be investigated.
- Modeling intracellular and cellular mechanisms of reference biological complex systems and proposing new experimental plans in an iterative way.

- Managing, processing and analyzing the workflow of image data obtained along different multidimensional microscopy modalities, at different scales with application in genome wide molecular screening, drug screening and medical diagnostic.

Solving novel "inverse problems", fusing multimodal and multiscale images and simulating dynamical processes are crucial as in many scientific fields. We hope the proposed integrated and innovative approaches and methods will help to guide future reasoning, modelling and experiments in cell biology.

\section{ACKNOWLEDGMENT}

The authors would like to thank the members of the Inria Serpico Project-Team and of the Bioimage Analysis unit (Institut Pasteur) for their inputs and contributions to some of the ideas and works presented in this paper. The authors thank the members of the "Space-time imaging of cellular dynamics of organelles and endomembranes" research team (UMR 144 CNRS Institut Curie) and S. Mukherjee (University of Virginia) for their contributions and assistance.

\section{REFERENCES}

[1] N. Chenouard et al., "An objective comparison of particle tracking methods," Nat. Meth., vol. 11, pp. 281-289, 2014.

[2] N. Bonnet, "Some trends in microscope image processing," Micron, vol. 35 , no. 8, pp. 645-654, 2004.

[3] M. Maska et al., "A benchmark for comparison of cell tracking algorithms," Bioinformatics, vol. 30, no. 11, pp. 1609-1617, 2014.

[4] F. Faugel, P. Smigielski, A. Brandenburg, and J. Fontaine, Biophotonics for Life Sciences and Medicine. Lausanne, Switzerland: Fontis Media SA, 2006.

[5] J. Rittscher, R. Machiraju, and S. T. C. Wong, Microscopic Image Analysis for Life Science Applications, 1st ed. Norwood, MA, USA: Artech House, 2008.

[6] R. F. Murphy, E. Meijering, and G. Danuser, "Molecular and cellular bioimaging," IEEE Trans. Image Process., vol. 14, no. 9, pp. 1233-1236, Sep. 2015.

[7] J. Kovacevic and R. F. Murphy, "Molecular and cellular bioimaging," IEEE Signal Process. Mag., vol. 23, no. 3, p. 19, May 2006.

[8] A. Munoz-Barrutia, J. Kovacevic, M. Kozubek, E. Meijering, and B. Parvin, "Quantitative bioimaging: Signal processing in light microscopy," IEEE Signal Process. Mag., vol. 32, no. 1, pp. 18-19, Jan. 2015.

[9] R. F. Murphy et al., "Focus on bioimage informatics," Nat. Meth., vol. 9, no. 7, pp. 629-742, 2012.

[10] E. Meijering, I. Smal, and G. Danuser, "Tracking in molecular bioimaging," IEEE Signal Process. Mag., vol. 23, no. 3, pp. 46-53, May 2006

[11] E. Meijering, O. Dzyubachyk, and I. Smal, "Methods for cell and particle tracking," Methods Enzym., vol. 504, no. 9, pp. 183-200, 2012.

[12] I. Smal and E. Meijering, "Quantitative comparison of multiframe data association techniques for particle tracking in time-lapse fluorescence microscopy," Med. Image Anal., vol. 24, no. 1, pp. 163-189, 2015.

[13] B. Rieger, R. Nieuwenhuizen, and S. Stallinga, "Image processing and analysis for single-molecule localization microscopy: Computation for nanoscale imaging," IEEE Signal Process. Mag., vol. 32, no. 1, pp. 49-57, Jan. 2015.

[14] R. J. Ober, A. Tahmasbi, S. Ram, L. Zhiping, and E. S. Ward, "Quantitative aspects of single-molecule microscopy: Information-theoretic analysis of single-molecule data," IEEE Signal Process. Mag., vol. 32, no. 1 , pp. $58-69$, Jan. 2015

[15] R. Delgado-Gonzalo, V. Uhlmann, D. Schmitter, and M. Unser, "Snakes on a plane: A perfect snap for bioimage analysis," IEEE Signal Process. Mag., vol. 32, no. 1, pp. 41-48, Jan. 2015.

[16] Z. Pincus and J. A. Theriot, "Comparison of quantitative methods for cell-shape analysis," J. Microscopy, vol. 227, pp. 140-156, 2007. 
[17] E. Meijering, "Neuron tracing in perspective," Cytometry A, vol. 77, no. 7, pp. 693-704, 2010.

[18] S. Bolte and F. Cordelières, "A guided tour into subcellular colocalization analysis in light microscopy," J. Microscopy, vol. 224, no. 3, pp. 213-232, 2006.

[19] T. Lagache, N. Sauvonnet, L. Danglot, and J.-C. Olivo-Marin, "Statistical analysis of molecule colocalization in bioimaging," Cytometry $A$, 2015.

[20] P. Sarder and A. Nehorai, "Deconvolution method for 3D fluorescence microscopy images," IEEE Signal Process. Mag., vol. 23, no. 3, pp. 32-45, May 2006

[21] I. Smal, M. Loog, W. J. Niessen, and E. Meijering, "Quantitative comparison of spot detection methods in fluorescence microscopy," IEEE Trans. Med. Imag., vol. 29, no. 2, pp. 282-301, Feb. 2010.

[22] V. Ljosa and A. E. Carpenter, "Introduction to the quantitative analysis of two-dimensional fluorescence microscopy images for cell-based screening,", PLoS Comp. Biol., vol. 5, no. 2, p. e1000603, 2009.

[23] T. Walter et al., "Visualization of image data from cells to organisms," Nat. Meth., vol. 7, pp. S26-S41, 2010.

[24] G. Danuser, "Computer vision in cell biology," Cell, vol. 147, no. 5, pp. 973-978, 2011.

[25] F. Long, J. Zhou, and H. Peng, "Visualization and analysis of 3D microscopi images," PLoS Comp. Biol., vol. 8, no. 6, p. e1002519, 2012.

[26] Z. Devaux, C. O. S. Sorzano, and S. Jonić, "chapter Processing of Transmission Electron Microscopy Images for Single-Particle Analysis of Macromolecular Complexes," in Laboratory Methods in Cell Biology. : Elsevier Inc., 2012, pp. 295-310.

[27] C. O. S. Sorzano et al., "chapter Semiautomatic, high-throughput, highresolution protocol for three-dimensional reconstruction of Single Particles in Electron Microscopy," in Nanoimaging: Methods and Protocols. Methods in Molecular Biology. New York, NY, USA: Humana, 2012, pp. 171-193.

[28] T. R. Shaikh, H. Gao, W. Baxter, F. J. Asturias, N. Boisset, A. Leith, and J. Frank, "SPIDER image processing for single-particle reconstruction of biological macromolecules from electron micrographs," Nat. Protocols, vol. 3, pp. 1941-1974, 2008.

[29] G. Tang, L. Peng, P. R. Baldwin, D. S. Mann, W. Jiang, I. Rees, and S. J. Ludtke, "EMAN2: An extensible image processing suite for electron microscopy," J. Struct. Biol., vol. 157, pp. 38-46, 2007.

[30] M. van Heel, G. Harauz, E. V. Orlova, R. Schmidt, and M. Schatz, "A new generation of the IMAGIC image processing system," J. Struct. Biol., vol. 116, pp. 17-24, 1996.

[31] C. O. S. Sorzano, R. Marabini, J. Velázquez-Muriel, J. R. Bilbao-Castro, S. H. W. Scheres, J. M. Carazo, and A. Pascual-Montano, "XMIPP: A new generation of an open-source image processing package for electron microscopy," J. Struct. Biol., vol. 148, pp. 194-204, 2004.

[32] S. H. W. Scheres, R. Núñez Ramírez, C. O. S. Sorzano, J. M. Carazo, and R. Marabini, "Image processing for electron microscopy singleparticle analysis using XMIPP," Nat. Protocols, vol. 3, pp. 977-990, 2008.

[33] J. M. de la Rosa-Trevín, J. Otón, R. Marabini, A. Zaldívar, J. Vargas, J. M. Carazo, and C. O. S. Sorzano, "XMIPP 3.0: An improved software suite for image processing in electron microscopy," J. Struct. Biol., vol. 184, no. 2, pp. 321-328, 2013.

[34] B. Heymann and D. Belnap, "BSOFT: Image processing and molecular modeling for electron microscopy," J. Struct. Biol., vol. 157, pp. 3-18, 2007.

[35] M. Hohn, G. Tang, G. Goodyear, P. R. Baldwin, Z. Huang, P. A. Penczek, C. Yang, R. M. Glaeser, P. D. Adams, and S. J. Ludtke, "SPARX, a new environment for cryo-EM image processing," $J$. Struct. Biol., vol. 157, no. 1, pp. 47-55, 2007.

[36] N. Grigorieff, "FREALIGN: High-resolution refinement of single particle structures," J. Struct. Biol., vol. 157, pp. 117-125, 2007.

[37] S. H. W. Scheres, "RELION: Implementation of a Bayesian approach to cryo-EM structure determination," J. Struct. Biol., vol. 180, no. 3, pp. 519-530, 2012.

[38] G. C. Lander et al., "APPION: An integrated, database-driven pipeline to facilitate EM image processing," J. Struct. Biol., vol. 166, pp. 95-102, 2009.

[39] R. Marabini, J. R. Macías, J. Vargas, A. Quintana, C. O. S. Sorzano, and J. M. Carazo, "On the development of three new tools for organizing and sharing information in three-dimensional electron microscopy," Acta Crystallogr. D Biol. Crystallogr., vol. 69, pp. 695-700, 2013, no. Pt 5.
[40] X. C. Bai, G. McMullan, and S. H. W. Scheres, "How cryo-EM is revolutionizing structural biology," Trends Biochem. Sci., vol. 40, pp. 49-57, 2014.

[41] A. Bartesaghi, A. Merk, M. J. Borgnia, J. L. S. Milne, and S. Subramaniam, "Prefusion structure of trimeric hiv-1 envelope glycoprotein determined by cryo-electron microscopy," Nat. Struct. Mol. Biol., vol. 20, no. 12, pp. 1352-1357, 2013.

[42] B. E. Bammes, R. H. Rochat, J. Jakana, D. H. Chen, and W. Chiu, "Direct electron detection yields cryo-EM reconstructions at resolutions beyond 3/4 nyquist frequency," J. Struct. Biol., vol. 177, no. 3, pp. 589-601, 2012.

[43] X. Li, P. Mooney, S. Zheng, C. R. Booth, M. B. Braunfeld, S. Gubbens, D. A. Agard, and Y. Cheng, "Electron counting and beam-induced motion correction enable near-atomic-resolution single-particle cryoEM," Nat. Meth., vol. 10, no. 6, pp. 584-590, 2013.

[44] H. Shigematsu and F. J. Sigworth, "Noise models and cryo-EM drift correction with a direct-electron camera," Ultramicroscopy, vol. 131, pp. 61-69, 2013.

[45] A. F. Brilot, J. Z. Chen, A. Cheng, J. Pan, S. C. Harrison, C. S. Potter, B. Carragher, R. Henderson, and N. Grigorieff, "Beam-induced motion of vitrified specimen on holey carbon film," J. Struct. Biol., vol. 177, no. 3, pp. 630-637, 2012.

[46] C. J. Russo and L. A. Passmore, "Electron microscopy. ultrastable gold substrates for electron cryomicroscopy," Science, vol. 346, no. 6215, pp. 1377-1380, 2014.

[47] M. G. Campbell et al., "Movies of ice-embedded particles enhance resolution in electron cryo-microscopy," Structure, vol. 20, no. 11, pp. $1823-1828,2012$

[48] X. C. Bai, I. S. Fernandez, G. McMullan, and S. H. W. Scheres, "Ribosome structures to near-atomic resolution from thirty thousand cryo-EM particles," Elife, vol. 2, p. e00461, 2013.

[49] S. H. W. Scheres, "Beam-induced motion correction for submegadalton cryo-EM particles," Elife, vol. 3, p. e03665, 2014.

[50] V. Abrishami, J. Vargas, X. Li, Y. Cheng, R. Marabini, C. O. S. Sorzano, and J. M. Carazo, "Alignment of direct detection device micrographs using a robust optical flow approach," J. Struct. Biol., vol. 189, no. 3, pp. 163-176, 2015.

[51] Z. Shang and F. J. Sigworth, "Hydration-layer models for cryo-EM image simulation," J. Struct. Biol., vol. 180, no. 1, pp. 10-16, 2012.

[52] M. Vulović, R. B. G. Ravelli, L. J. van Vliet, A. J. Koster, I. Lazić, U. Lücken, H. Rullgård, O. Öktem, and B. Rieger, "Image formation modeling in cryo-electron microscopy," J. Struct. Biol., vol. 183, no. 1, pp. 19-32, 2013.

[53] M. Vulović, L. M. Voortman, L. J. van Vliet, and B. Rieger, "When to use the projection assumption and the weak-phase object approximation in phase contrast cryo-EM," Ultramicroscopy, vol. 136C, pp. 61-66, 2013

[54] R. F. Egerton, "Control of radiation damage in the TEM," Ultramicroscopy, vol. 127, pp. 100-108, 2013.

[55] J. Vargas, J. Otón, R. Marabini, S. Jonic, J. M. de la Rosa-Trevín, J. M. Carazo, and C. O. S. Sorzano, "Fastdef: Fast defocus and astigmatism estimation for high-throughput transmission electron microscopy," $J$. Struct. Biol., vol. 181, no. 2, pp. 136-148, 2013.

[56] V. Abrishami, A. Zaldívar-Peraza, J. M. de la Rosa-Trevín, J. Vargas, J. Otón, R. Marabini, Y. Shkolnisky, J. M. Carazo, and C. O. S. Sorzano, "A pattern matching approach to the automatic selection of particles from low-contrast electron micrographs," Bioinformatics, vol. 29, no. 19, pp. 2460-2468, 2013.

[57] M. Shatsky, P. Arbelaez, B. G. Han, D. Typke, S. E. Brenner, J. Malik, and R. M. Glaeser, "Automated particle correspondence and accurate tilt-axis detection in tilted-image pairs," J. Struct. Biol., vol. 187, no. 1, pp. 66-75, 2014.

[58] R. Norousi, S. Wickles, C. Leidig, T. Becker, V. J. Schmid, R. Beckmann, and A. Tresch, "Automatic post-picking using mappos improves particle image detection from cryo-EM micrographs," J. Struct. Biol., vol. 182 , no. 2 , pp. 59-66, 2013.

[59] J. Vargas, V. Abrishami, R. Marabini, J. M. de la Rosa-Trevín, A. Zaldivar, J. M. Carazo, and C. O. S. Sorzano, "Particle quality assessment and sorting for automatic and semiautomatic particle-picking techniques," J. Struct. Biol., vol. 183, no. 3, pp. 342-353, 2013.

[60] Z. Zhao and A. Singer, "Rotationally invariant image representation for viewing direction classification in cryo-EM," J. Struct. Biol., vol. 186, no. 1, pp. 153-166, 2014.

[61] Z. Yang, J. Fang, J. Chittuluru, F. J. Asturias, and P. A. Penczek, "Iterative stable alignment and clustering of 2D transmission electron microscope images," Structure, vol. 20, no. 2, pp. 237-247, 2012. 
[62] C. O. S. Sorzano et al., "Outlier detection for single particle analysis in electron microscopy," in Proc. Int. Work-Conf. Bioinf. Biomed. Eng. (IWBBIO), Granada, Spain, 2014, pp. 950-959.

[63] Z. Zhao and A. Singer, "Fourier-bessel rotational invariant eigenimages," J. Opt. Soc. Amer. A, vol. 30, pp. 871-877, 2012.

[64] D. Elmlund and H. Elmlund, "Simple: Software for ab initio reconstruction of heterogeneous single-particles," J. Struct. Biol., vol. 180, no. 3, pp. 420-427, 2012.

[65] D. Giannakis, P. Schwander, and A. Ourmazd, "The symmetries of image formation by scattering. i. Theoretical framework," Opt. Exp., vol. 20, no. 12, pp. 12799-12826, 2012.

[66] Y. Shkolnisky and A. Singer, "Viewing direction estimation in cryo-EM using synchronization," SIAM J. Imag. Sci., vol. 5, no. 3, pp. 1088-1110, 2012

[67] H. Elmlund, D. Elmlund, and S. Bengio, "Prime: Probabilistic initial 3D model generation for single-particle cryo-electron microscopy," Structure, vol. 21, no. 8, pp. 1299-1306, 2013.

[68] D. Lyumkis, S. Vinterbo, C. S. Potter, and B. Carragher, "Optimod-An automated approach for constructing and optimizing initial models for single-particle electron microscopy," J. Struct. Biol., vol. 184, no. 3, pp. 417-426, 2013.

[69] L. Wang, A. Singer, and Z. Wen, "Orientation determination of cryo-EM images using least unsquared deviations," SIAM J. Imag. Sci., vol. 6, no. 4, pp. 2450-2483, 2013.

[70] J. Vargas, A. L. Álvarez-Cabrera, R. Marabini, J. M. Carazo, and C. O. S. Sorzano, "Efficient initial volume determination from electron microscopy images of single particles," Bioinformatics, vol. 30, pp. 2891-2898, 2014

[71] C. O. S. Sorzano, J. Vargas, J. M. de la Rosa-Trevín, J. Otón, A. L. Álvarez-Cabrera, V. Abrishami, E. Sesmero, R. Marabini, and J. M. Carazo, "A statistical approach to the initial volume problem in single particle analysis by electron microscopy," J. Struct. Biol., vol. 189, no. 3, pp. 213-219, 2015.

[72] L. Wang, Y. Shkolnisky, and A. Singer, "A Fourier-based approach for iterative 3D reconstruction from cryo-EM images," arXiv Preprint arXiv: $1307.5824,2013$.

[73] A. Kucukelbir, F. J. Sigworth, and H. D. Tagare, "A Bayesian adaptive basis algorithm for single particle reconstruction," J. Struct. Biol., vol. 179, no. 1, pp. 56-67, 2012.

[74] B. Chen, B. Shen, and J. Frank, "Particle migration analysis in iterative classification of cryo-EM single-particle data," J. Struct. Biol., vol. 188, no. 3, pp. 267-273, 2014.

[75] S. H. W. Scheres, "A Bayesian view on cryo-EM structure determination,” J. Molec. Biol., vol. 415, no. 2, pp. 406-418, 2012.

[76] D. Lyumkis, A. F. Brilot, D. L. Theobald, and N. Grigorieff, "Likelihood-based classification of cryo-EM images using frealign," J. Struct. Biol., vol. 183, no. 3, pp. 377-388, 2013.

[77] A. Dashti, P. Schwander, R. Langlois, R. Fung, W. Li, A. Hosseinizadeh, H. Y. Liao, J. Pallesen, G. Sharma, V. A. Stupina, A. E. Simon, J. D. Dinman, J. Frank, and A. Ourmazd, "Trajectories of the ribosome as a Brownian nanomachine," in Proc. Nat. Acad. Sci. USA, 2014, vol. 111, no. 49, pp. 17492-17497.

[78] J. Qiyu, I. Grama, and Q. Liu, "A new Poisson noise filter based on weights optimization,” J. Sci. Comput., vol. 58, no. 3, pp. 548-573, 2014.

[79] G. Katsevich, A. Katsevich, and A. Singer, "Covariance matrix estimation for the cryo-EM heterogeneity problem," arXiv Preprint arXiv:1309.1737, 2013.

[80] Q. Wang, T. Matsui, T. Domitrovic, Y. Zheng, P. C. Doerschuk, and J. E. Johnson, "Dynamics in cryo em reconstructions visualized with maximum-likelihood derived variance maps," J. Struct. Biol., vol. 181, no. 3, pp. 195-206, Mar. 2013.

[81] J. Andén, E. Katsevich, and A. Singer, "Covariance estimation using conjugate gradient for 3D classification in cryo-EM," arXiv Preprint arXiv:1412.0985, 2014.

[82] R. Henderson et al., "Outcome of the first electron microscopy validation task force meeting," Structure, vol. 20, no. 2, pp. 205-214, 2012.

[83] P. Cossio and G. Hummer, "Bayesian analysis of individual electron microscopy images: Towards structures of dynamic and heterogeneous biomolecular assemblies," J. Struct. Biol., vol. 184, no. 3, pp. 427-437, 2013.

[84] C. J. Russo and L. A. Passmore, "Robust evaluation of 3D electron cryomicroscopy data using tilt-pairs," J. Struct. Biol., vol. 187, no. 2, pp. 112-118, 2014.

[85] J. Vargas, Otón, R. Marabini, J. M. Carazo, and C. O. S. Sorzano, "Statistical analysis of 3D electron cryomicroscopy data without using tilt-pairs," Scientific Reports, 2015, to be published.
[86] A. Kucukelbir, F. J. Sigworth, and H. D. Tagare, "Quantifying the local resolution of cryo-EM density maps," Nat. Meth., vol. 11, pp. 63-65, 2014.

[87] A. Biswas, D. Si, K. Al Nasr, D. Ranjan, M. Zubair, and J. He, "Improved efficiency in cryo-EM secondary structure topology determination from inaccurate data," J. Bioinf. Comput. Biol., vol. 10, no. 3, p. 1242006, 2012.

[88] D. Si, S. Ji, K. Al Nasr, and J. He, "A machine learning approach for the identification of protein secondary structure elements from electron cryo-microscopy density maps," Biopolymers, vol. 97, no. 9, pp. 698-708, 2012.

[89] K. Al Nasr, D. Ranjan, M. Zubair, L. Chen, and J. He, "Solving the secondary structure matching problem in cryo-EM de novo modeling using a constrained k-shortest path graph algorithm," IEEE/ACM Trans. Comput. Biol. Bioinf., vol. 11, no. 2, pp. 419-430, 2014.

[90] M. Rey et al., "Mass spec studio for integrative structural biology," Structure, vol. 22, no. 10, pp. 1538-1548, 2014.

[91] J. Velàsquez-Muriel, K. Lasker, D. Russel, J. Phillips, B. M. Webb, D. Schneidman-Duhovny, and A. Sali, "Assembly of macromolecular complexes by satisfaction of spatial restraints from electron microscopy images," Proc. Nat. Acad. Sci. USA, vol. 109, no. 46, pp. 18821-18826, 2012.

[92] M. S. Chapman, A. Trzynka, and B. K. Chapman, "Atomic modeling of cryo-electron microscopy reconstructions-Joint refinement of model and imaging parameters," J. Struct. Biol., vol. 182, no. 1, pp. 10-21, 2013.

[93] J. Esquivel-Rodríguez and D. Kihara, "Computational methods for constructing protein structure models from 3D electron microscopy maps," J. Struct. Biol., vol. 184, no. 1, pp. 93-102, 2013.

[94] J. R. López-Blanco and P. Chacón, "iMODFIT: Efficient and robust flexible fitting based on vibrational analysis in internal coordinates," $J$. Struct. Biol., vol. 184, no. 2, pp. v261-270, 2013.

[95] A. P. Pandurangan, S. Shakeel, S. J. Butcher, and M. Topf, “Combined approaches to flexible fitting and assessment in virus capsids undergoing conformational change," J. Struct. Biol., vol. 185, no. 3, pp. 427-439, 2014

[96] E. Villa and K. Lasker, "Finding the right fit: Chiseling structures out of cryo-electron microscopy map," Curr. Opin. Struct. Biol., vol. 25, pp. 118-125, 2014.

[97] R. Nogales-Cadenas et al., "3DEM loupe: Analysis of macromolecular dynamics using structures from electron microscopy," Nucleic Acids Res., vol. 41, pp. W363-W367, 2013, no. Web Server issue.

[98] Y. Chen, S. Pfeffer, T. Hrabe, J. M. Schuller, and F. Förster, "Fast and accurate reference-free alignment of subtomograms," J. Struct. Biol., vol. 182, pp. 235-245, 2013

[99] O. Kuybeda, G. A. Frank, A. Bartesaghi, M. Borgnia, S. Subramaniam, and G. Sapiro, "A collaborative framework for 3D alignment and classification of heterogeneous subvolumes in cryo-electron tomography," J. Struct. Biol., vol. 181, no. 2, pp. 116-127, 2013.

[100] M. Shatsky, P. Arbelaez, R. M. Glaeser, and S. E. Brenner, "Optimal and fast rotational alignment of volumes with missing data in Fourier space," J. Struct. Biol., vol. 184, no. 2, pp. 345-347, 2013.

[101] Y. Chen, S. Pfeffer, J. J. Fernández, C. O. S. Sorzano, and F. Förster, "Autofocused 3D classification of cryoelectron subtomograms," Structure, vol. 22, no. 10, pp. 1528-1537, 2014.

[102] Z. Yu and A. S. Frangakis, "M-free: Scoring the reference bias in subtomogram averaging and template matching," J. Struct. Biol., vol. 187, no. 1, pp. 10-19, 2014.

[103] L. M. Voortman et al., "Quantifying resolution limiting factors in subtomogram averaged cryo-electron tomography using simulations," J. Struct. Biol., vol. 187, no. 2, pp. 103-111, 2014.

[104] J. A. G. Briggs, "Structural biology in situ-The potential of subtomogram averaging," Curr. Opin. Struct. Biol., vol. 23, no. 2, pp. 261-267, 2013.

[105] A. Bartesaghi, F. Lecumberry, G. Sapiro, and S. Subramaniam, "Protein secondary structure determination by constrained single-particle cryo-electron tomography," Structure, vol. 20, no. 12, pp. 2003-2013, 2012.

[106] B. Goris, T. Roelandts, K. J. Batenburg, H. H. Mezerji, and S. Bals, "Advanced reconstruction algorithms for electron tomography: From comparison to combination," Ultramicroscopy, vol. 127, pp. 40-47, 2013.

[107] C. Messaoudi, N. Aschman, M. Cunha, T. Oikawa, C. O. S. Sorzano, and S. Marco, "Three-dimensional chemical mapping by Eftem-Tomoj including improvement of SNR by PCA and art reconstruction of volume by noise suppression," Microsc. Microanal., vol. 19, no. 6, pp. 1669-1677, 2013. 
[108] L. Kovacik, S. Kereïche, J. L. Höög, P. Juda, P. Matula, and I. Raska, "A simple Fourier filter for suppression of the missing wedge ray artefacts in single-axis electron tomographic reconstructions," J. Struct. Biol., 2014, to be published.

[109] M. Maiorca, C. Millet, E. Hanssen, B. Abbey, E. Kazmierczak, and L. Tilley, "Local regularization of tilt projections reduces artifacts in electron tomography," J. Struct. Biol., vol. 186, no. 1, pp. 28-37, 2014.

[110] R. Danev, B. Buijsse, M. Khoshouei, J. M. Plitzko, and W. Baumeister, "Volta potential phase plate for in-focus phase contrast transmission electron microscopy," in Proc. Nat. Acad. Sci. USA, 2014, vol. 111, no. 44 , pp. $15635-15640$.

[111] M. Chalfie, Y. Tu, G. Euskirchen, W. W. Ward, and D. C. Prasher, "Green fluorescent protein as a marker for gene expression," Science, vol. 263, no. 5148, pp. 802-805, 1994.

[112] M. A. Digman and E. Gratton, "Lessons in fluctuation correlation spectroscopy,” Ann. Rev. Phys. Chem., vol. 62, pp. 645-668, 2011.

[113] S. W. Hell and J. Wichmann, "Breaking the diffraction resolution limit by stimulated emission," Opt. Lett., vol. 19, no. 11, pp. 780-782, 1994.

[114] M. J. Rust, M. Bates, and X. Zhuang, "Sub-diffraction-limit imaging by stochastic optical reconstruction microscopy (STORM)," Nat. Meth., vol. 3, pp. 793-796, 2006.

[115] M. Heilemann, E. Margeat, R. Kasper, M. Sauer, and P. Tinnefeld, "Carbocyanine dyes as efficient reversible single-molecule optical switch,” J. Amer. Chem. Soc., vol. 127, pp. 3801-3806, 2005.

[116] E. Betzig et al., "Imaging intracelluar fluorescent proteins at nanometer resolution," Science, vol. 313, pp. 1642-1645, 2006.

[117] S. T. Hess, T. P. K. Giriajan, and M. D. Mason, "Ultra-high resolution imaging by fluorescence photoactivation localization microscopy," Biophys. J., vol. 91, pp. 4258-4272, 2006.

[118] M. G. L. Gustafsson, "Surpassing the lateral resolution limit by a factor of two using structured illumination microscopy," J. Microscopy, vol. 198, pp. 82-87, 2000.

[119] M. G. L. Gustafsson et al., "3D resolution doubling in wide-field fluorescence microscopy by structured illumination," Biophys. J., vol. 94, pp. 4957-4970, 2008.

[120] H. Shroff, C. G. Galbraith, J. A. Galbraith, and E. Betzig, "Live-cell photoactivated localization microscopy of nanoscale adhesion dynamics," Nat. Meth., vol. 5, pp. 417-423, 2008.

[121] D. Li et al., "Extended-resolution structured illumination imaging of endocytic and cytoskeletal dynamics spatiotemporal resolution," Science, vol. 349, no. 6251, p. aab3500, 2015.

[122] B.-C. Chen et al., "Lattice light-sheet microscopy: Imaging molecules to embryos at high spatiotemporal resolution," Science, vol. 346, no. 6208, p. $1257998,2014$.

[123] M. van't Hoff, V. de Sars, and M. Oheim, "A programmable light engine for quantitative single molecule TIRF and HILO imaging," Opt. Exp., vol. 16, no. 22, pp. 18495-18504, 2008.

[124] A. Sharonov and R. M. Hochstrasser, "Wide-field subdiffraction imaging by accumulated binding of diffusing probes," in Proc. Nat. Acad. Sci. USA, 2006, vol. 103, pp. 18911-18916.

[125] J. Boulanger et al., "Fast high-resolution 3D total internal reflection fluorescence microscopy by incidence angle scanning and azimuthal averaging," in Proc. Nat. Acad. Sci. USA., 2014, vol. 111, no. 48, pp. 17164-17169.

[126] A. Sartori, R. Gatz, F. Beck, A. Rigort, W. Baumeister, and J. M. Plitzko, "Correlative microscopy: Bridging the gap between fluorescence light microscopy and cryo-electron tomography," J. Struct. Biol., vol. 160, pp. 135-145, 2007.

[127] X. Heiligenstein et al., "The CryoCapsule: Simplifying correlative light to electron microscopy," Traffic, vol. 15, no. 6, pp. 700-716, 2014.

[128] C. Van Rijnsoever, V. Oorschot, and J. Klumperman, "Correlative light-electron microscopy (CLEM) combining live-cell imaging and immunolabeling of ultrathin cryosections," Nat. Meth., vol. 5, pp. 973-980, 2008.

[129] B. S. Padman, M. Bach, and G. Ramm, "An improved procedure for subcellular spatial alignment during live-cell CLEM," PLoS One, vol. 9, p. e95967, 2014.

[130] V. W. Wells, P. Viola, H. Atsumi, S. Nakajima, and R. Kikinis, "Multi-modal registration by maximization of mutual information," Med. Image Anal., vol. 91, no. 1, pp. 35-51, 1996.

[131] A. Roche, G. Malandain, and N. Ayache, "Unifying maximum likelihood approaches in medical image registration," Int. J. Imag. Syst. Technol., vol. 11, pp. 71-80, 2000.

[132] G. Vicidomini et al., "High data output and automated 3D correlative light-electron microscopy method," Traffic, vol. 9, pp. 1828-1838, 2008.
[133] P. M. Carlton et al., "Fast live simultaneous multiwavelength four-dimensional optical microscopy," in Proc. Nat. Acad. Sci. USA, 2010, vol. 107 , no. 37 , pp. 16016-16022.

[134] J. Fink et al., "External forces control mitotic spindle positioning," Nat. Cell Biol., vol. 13, no. 7, pp. 771-778, 2011.

[135] R. M. Parton et al., "A par-1dependent orientation gradient of dynamic microtubules directs posterior cargo transport in the drosophila oocyte," J. Cell Biol., vol. 194, no. 1, pp. 121-135, 2011.

[136] Y. Luo, Y. Zhan, and J. H. Keen, "Arf6 regulation of gyrarting-clathrin," Traffic, vol. 14, pp. 97-106, 2012.

[137] J. Boulanger, C. Kervrann, P. Bouthemy, P. Elbau, J.-B. Sibarita, and J. Salamero, "Patch-based non-local functional for denoising fluorescence microscopy image sequences," IEEE Trans. Med. Imag., vol. 29, no. 2, pp. 442-454, 2010.

[138] A. E. Saliba et al., "Microfluidic sorting and high content multimodal typing of cancer cells in self-assembled magnetic arrays," in Proc. Nat. Acad. Sci. USA, 2010, vol. 107, no. 33, pp. 14524-14529.

[139] S. Dokudovskaya et al., "A conserved coatomer-related complex containing Sec13 and Seh1 dynamically associates with the vacuole in Saccharomyces cerevisiae," Mol. Cell Proteomics, 2011.

[140] A. Matsuda, L. Shao, J. Boulanger, C. Kervrann, P. M. Carlton, P. Kner, E. Brandlund, D. Agard, and J. W. Sedat, "Condensed mitotic chromosome structure at nanometer resolution using palm and EGFP-histones," PLoS One, vol. 5, no. 9, p. e12768, 2010.

[141] A. Foi, M. Trimeche, V. Katkovnik, and K. Egiazarian, "Practical Poissonnian-Gaussian noise modeling and fitting for single-image raw-data," IEEE Trans. Image Process., vol. 17, no. 10, pp. 1737-1754, 2008.

[142] F. Starck, J.-L. Murtagh, and A. Bijaoui, Image Processing and Data Analysis. Cambridge, U.K.: Cambridge Univ. Press, 1998.

[143] B. Zhang, J. Fadili, and J. Starck, "Wavelets, ridgelets, and curvelets for Poisson noise removal," IEEE Trans. Image Process., vol. 51, no. 1, pp. 1093-1108, 2008 .

[144] M. Makitalo and A. Foi, "Optimal inversion of the generalized Anscombe transformation for Poisson-Gaussian noise," IEEE Trans. Image Process., vol. 2, no. 1, pp. 91-103, Jan. 2013.

[145] F. J. Anscombe, "The transformation of Poisson, binomial and negative-binomial data," Biometrika, vol. 35, pp. 246-254, 1948.

[146] M. Fisz, "The limiting distribution of a function of two independent random variables and its statistical application," Coll. Math., vol. 3, pp. 138-146, 1955.

[147] B. Zhang, M. J. Fadili, J.-L. Starck, and J.-C. Olivo-Marin, "Multiscale variance-stabilizing transform for mixed-Poisson-Gaussian processes and its applications in bioimaging," in Proc. IEEE Int. Conf. Image Process. (ICIP), San Antonio, TX, USA, 2007, vol. 6, pp. 233-236.

[148] V. O. O'Connor and D. Phillips, Time-correlated Single Photon Counting. London, U.K.: Academic, 1984.

[149] A. Chessel, F. Waharte, J. Salamero, and C. Kervrann, "A maximum likelihood method for lifetime estimation in photon counting-based fluorescence lifetime imaging microscopy," in Proc. Eur. Signal Process. Conf. (EUSIPCO), Marrakech, Morocco, 2013, pp. 1-5.

[150] M. A. T. Figueiredo and J. M. Bioucas-Dias, "Restoration of Poissonian images using alternating direction optimization," IEEE Trans. Image Process., vol. 19, no. 12, pp. 3133-3145, Dec. 2010.

[151] A. Jezierska, C. Chaux, J.-C. Pesquet, and H. Talbot, "Poisson-gaussian noise parameter estimation in fluorescence microscopy," in Proc. IEEE Int. Symp. Biomed. Imag. (ISBI), Barcelona, Spain, 2012, pp. $1663-1666$.

[152] C. Jezierska, A. Chaux, J.-C. Pesquet, G. Talbot, and H. Engle, “An em approach for time-variant Poisson-Gaussian model parameter estimation," IEEE Trans. Signal Process., vol. 62, no. 1, pp. 17-30, Jan. 2014.

[153] W. Richardson, "Bayesian-based iterative method of image restoration,” J. Opt. Soc. Amer., vol. 62, pp. 55-59, 1972.

[154] I. C. Rodrigues and J. M. R. Sanchez, "Convex total variation denoising of Poisson fluorescence confocal images with anisotropic filtering," IEEE Trans. Image Process., vol. 20, no. 1, pp. 146-160, Jan. 2011.

[155] A. Buades, B. Coll, and J. M. Morel, "A review of image denoising methods, with a new one," SIAM J. Multiscale Model. Simul., vol. 4, no. 2, pp. 490-530, 2005.

[156] A. de Decker, J. A. Lee, and M. Verlysen, "Variance stabilizing transformations in patch-based bilateral filters for poisson noise image denoising," in Proc. IEEE Eng. Med. Biol. Soc. (EMBS), Minneapolis, MN, USA, 2009, pp. 3673-3676.

[157] A. Bindilatti and N. Mascarenhas, "Patch reprojections for Non-Local methods,” Signal Process. Lett., vol. 20, no. 11, pp. 1010-101, 2013. 
[158] J. Salmon, C. A. Deledalle, R. Willett, and Z. T. Harmany, "Poisson noise reduction with non-local PCA," in Proc. IEEE Int. Conf. Acoust., Speech, Signal Process. (ICASSP), Kyoto, Japan, 2012, pp. 1109-1112.

[159] R. Giryes and M. Elad, "Sparsity based poisson denoising," in Proc. Elect. Electron. Eng. Israel (IEEEI'12), Eilat, Israel, 2012, pp. 1-5.

[160] C.-A. Deledalle, F. Tupin, and L. Denis, "Poisson NL-means: Unsupervised non local means for Poisson noise," in Proc. IEEE Int. Conf. Image Process. (ICIP), Hong Kong, China, 2010, pp. 801-804.

[161] P. Coupé, M. Munz, J. V. Manjón, E. S. Ruthazer, and D. L. Collins, "A CANDLE for a deeper in vivo insight," Med. Image Anal., vol. 16, no. 4, pp. 849-864, 2012.

[162] P. Roudot, C. Kervrann, J. Boulanger, and F. Waharte, "Noise modeling for intensified camera in fluorescence imaging: Application to image denoising," in Proc. IEEE Int. Symp. Biomed. Imag. (ISBI), San Francisco, CA, USA, 2013, pp. 600-603.

[163] P. Roudot, C. Kervrann, and F. Waharte, "Lifetime estimation on moving sub-cellular objects in frequency domain FLIM imaging," $J$. Opt. Soc. Amer. A, vol. 32, no. 10, pp. 1821-1835, 2015.

[164] D. L. Donoho, "De-noising by soft-thresholding," IEEE Trans. Inf Theory, vol. 41, no. 3, pp. 613-627, May 1995.

[165] R. M. Willett and R. D. Nowak, "Platelets: A multiscale approach for recovering edges and surfaces in photon-limited medical imaging," IEEE Trans. Med. Imag., vol. 22, no. 3, pp. 332-350, Mar. 2003.

[166] F. Luisier, C. Vonesch, T. Blu, and M. Unser, "Fast haar-wavelet denoising of multidimensional fluorescence microscopy data," in Proc. IEEE Int. Symp. Biomed. Imag. (ISBI), Boston, MA, USA, 2009, pp. 310-313.

[167] F. Luisier, C. Vonesch, T. Blu, and M. Unser, "Fast interscale wavelet denoising of Poisson-corrupted images," Signal Process., vol. 90, no. 2, pp. 415-427, 2010 .

[168] F. Luisier, T. Blu, and M. Unser, "Image denoising in mixed Poisson-Gaussian noise," IEEE Trans. Image Process., vol. 20, no. 3, pp. 696-708, Mar. 2011

[169] Y. Le Montagner, E. D. Angelini, and J.-C. Olivo-Marin, "An unbiased risk estimator for image denoising in the presence of mixed Poisson-Gaussian noise," IEEE Trans. Image Process., vol. 23, no. 3 , pp. 1255-1268, Mar. 2014

[170] M. Marim, E. D. Angelini, and J.-C. Olivo-Marin, "A compressed sensing approach for biological microscopic image processing," in Proc. IEEE Int. Symp. Biomed. Imag. (ISBI), Boston, MA, USA, 2009, pp. 1374-1377.

[171] V. Studer, J. Bobin, M. Chahid, E. Candes, and M. Dahan, "Compressive fluorescence microscopy for biological and hyperspectral imaging," in Proc. Nat. Acad. Sci. USA, 2012, vol. 109, no. 26, pp. 1679-1687.

[172] S. Delpretti, F. Luisier, S. Ramani, T. Blu, and M. Unser, "Multiframe sure-let denoising of timelapse fluorescence microscopy images," in Proc. IEEE Int. Symp. Biomed. Imag. (ISBI), Paris, France, 2008, pp. $149-152$.

[173] J.-B. Sibarita, "Deconvolution microscopy," Microsc. Tech., Adv. Biochem. Eng., vol. 95, pp. 201-243, 2005.

[174] A. Griffa, N. Garin, and D. Sage, "comparison of deconvolution software in 3D microscopy: A user point of view-Part 1," G. I. T. Imag. Microsc., vol. 12, no. 1, pp. 43-45, 2010.

[175] A. Griffa, N. Garin, and D. Sage, "comparison of deconvolution software in 3D microscopy: A user point of view-Part 2," G. I. T. Imag. Microsc., vol. 12, no. 1, pp. 41-43, 2010.

[176] W. H. Richardson, "Bayesian-based iterative method of image restoration," J. Opt. Soc. Amer. A, vol. A 62, pp. 55-59, 1972.

[177] L. B. Lucy, "An iterative technique for the rectification of observed distributions," Astronom. J., vol. 79, pp. 745-754, 1974.

[178] C. Vonesch and M. Unser, "A fast thresholded Landweber algorithm for wavelet-regularized multidimensional deconvolution," IEEE Trans. Image Process., vol. 17, no. 4, pp. 539-549, Apr. 2008.

[179] F. Soulez, "A learn 2D, apply 3D method for 3D deconvolution microscopy," in Proc. IEEE Int. Symp. Biomed. Imag. (ISBI), Beijing, China, 2014, pp. 1075-1078.

[180] S. Lefkimmiatis and M. Unser, "Poisson image reconstruction with Hessian Schatten-norm regularization," IEEE Trans. Image Process., vol. 22, no. 11, pp. 4314-4327, Nov. 2013.

[181] M. Arigovindan et al., "High-resolution restoration of 3D structures from widefield images with extreme low signal-to-noise-ratio," in Proc. Nat. Acad. Sci. USA, 2013, vol. 110, no. 43, pp. 17344-17349.

[182] F. Orieux, E. Sepulveda, V. Loriette, B. Dubertret, and J.-C. OlivoMarin, "Bayesian estimation for optimized structured illumination microscopy," IEEE Trans. Image Process., vol. 21, no. 2, pp. 601-614, Feb. 2012.
[183] J. Min, C. Vonesch, H. Kirshner, L. Carlini, N. Olivier, S. Holden, S. Manley, J. C. Ye, and M. Unser, "FALCON: Fast and unbiased reconstruction of high-density super-resolution microscopy data," Sci. Rep., vol. 4, no. 4577, pp. 1-9, 2014.

[184] U. Koethe, F. Herrmannsdoerfer, I. Kats, and F. A. Hamprecht, "Simplestorm: A fast, self-calibrating reconstruction algorithm for localization microscopy," Histochem. Cell Biol., vol. 141, no. 6, pp. 613-627, 2014.

[185] J. Byun, M. R. Verardo, B. Sumengen, G. P. Lewis, B. S. Manjunath, and S. K. Fisher, "Automated tool for the detection of cell nuclei in digital microscopic images: Application to retinal images," Molecular Vis. , vol. 12, pp. 949-960, 2006.

[186] C. Jackson, E. Glory, R. F. Murphy, and J. Kovačević, "Model building and intelligent acquisition with application to protein subcellular location classification," Bioinformatics, vol. 27, no. 13, pp. 1854-1859, 2011.

[187] I. F. Sbalzarini and P. Koumoutsakos, "Feature point tracking and trajectory analysis for video imaging in cell biology," J. Struct. Biol., vol. 151, no. 2, pp. 182-195, 2005

[188] K. Jaqaman, D. Loerke, M. Mettlen, H. Kuwata, S. Grinstein, S. L. Schmid, and G. Danuser, "Robust single-particle tracking in live-cell time-lapse sequences," Nat. Meth., vol. 5, no. 5, pp. 695-702, 2008.

[189] D. Sage, F. R. Neumann, H. Hediger, S. M. Gasser, and M. Unser, "Automatic tracking of individual fluorescence particles: Application to the study of chromosome dynamics," IEEE Trans. Image Process., vol. 14, no. 9, pp. 1372-1383, Sep. 2005.

[190] J. Boulanger, A. Gidon, C. Kervrann, and J. Salamero, "A patch-based method for repetitive and transcient event detection in fluorescence imaging," PLoS One, vol. 5, no. 10, p. e13190, 2010.

[191] L. Sironi, J. Solon, C. Conrad, T. U. Mayer, D. Brunner, and J. Ellenberg, "Automatic quantification of microtubule dynamics enables RNAi-screening of new mitotic spindle regulators," Cytoskeleton, vol. 68 , no. 5, pp. 266-278, 2011

[192] A. Basset, P. Bouthemy, J. Boulanger, J. Salamero, and C. Kervrann, "Localization and classification of membrane dynamics in TIRF microscopy image sequences," in Proc. IEEE Int. Symp. Biomed. Imag. (ISBI), Beijing, China, 2014, pp. 830-833.

[193] R. Sebastian et al., "Spatio-temporal analysis of constitutive exocytosis in epithelial cells," IEEE Trans. Comp. Biol. Bioinf., vol. 3, pp. 17-32, 2006.

[194] K. Li and T. Kanade, "Nonnegative mixed-norm preconditioning for microscopy image segmentation," in Proc. Inf. Process. Med. Imag. (IPMI), Williamsburg, VA, USA, 2009, vol. 5636, pp. 362-373.

[195] D. Hu, P. Sarder, P. Ronhovde, S. Orthaus, S. Achilefu, and Z. Nussinov, "Automatic segmentation of fluorescence lifetime microscopy images of cells using multiresolution community detection-A first study," J. Microscopy, vol. 253, no. 1, pp. 54-64, 2013.

[196] G. Li, T. Liu, A. Tarokh, J. Nie, L. Guo, A. Mara, S. Holley, and S. T. C. Wong, "3D cell nuclei segmentation based on gradient flow tracking," BMC Cell Biol., vol. 40, no. 8, 2007.

[197] N. Phansalkar, S. More, A. Sabale, and M. Joshi, "Adaptive loca thresholding for detection of nuclei in diversity stained cytology images," in Proc. Int. Conf. Commun. Signal Process., Kerala, India, 2011, pp. 218-220.

[198] K. Bashar, K. Komatsu, T. Fujimori, and T. J. Kobayashi, "Automatic extraction of nuclei centroids of mouse embryonic cells from fluorescence microscopy images," PLoS One, vol. 7, no. 5, p. e35550, 2012.

[199] P. Ruusuvuori, T. Äijö, S. Chowdhury, C. Garmendia-Torres, J Selinummi, M. Birbaumer, A. M. Dudley, L. Pelkmans, and O. Yli-Harja, "Evaluation of methods for detection of fluorescence labeled subcellular objects in microscope images," BMC Bioinf., vol. 11 , no. 1,2010

[200] N. Otsu, "A threshold selection method from gray-level histograms," IEEE Trans. Syst., Man, Cybern., vol. SMC-9, no. 1, pp. 62-66, Jan. 1979.

[201] J. N. Kapur, P. K. Sahoo, and A. K. C. Wong, "A new method for gray-level picture thresholding using the entropy of the histogram," Comp. Vis., Graphics, Image Process., vol. 29, no. 3, pp. 273-285, 1985.

[202] P. K. Sahoo, D. W. Slaaf, and T. A. Albert, "Threshold selection using a minimal histogram entropy difference," Opt. Eng., vol. 36, no. 7, pp. 1976-1981, 1997

[203] A. Dempster, N. Laird, and D. Rubin, "Maximum likelihood from incomplete data via the EM algorithm," J. Roy. Statist. Soc. Ser. B, vol. 39 , no. 1 , pp. $1-38,1977$ 
[204] A. Tsai, W. M. Wells, S. K. Warfield, and A. S. Willsky, "An EM algorithm for shape classification based on level sets," Med. Image Anal., vol. 9, no. 5, pp. 491-502, 2005.

[205] P. K. Sahoo, S. Soltani, and A. K. C. Wong, "A survey of thresholding techniques," Comp. Vis., Graphics, Image Process., vol. 41, no. 2, pp. 233-260, 1988

[206] M. Sezgin and B. Sankur, "Survey over image thresholding techniques and quantitative performance evaluation," J. Electron. Imag., vol. 13, no. 1, pp. 146-168, 2004.

[207] D. Thomann, D. R. Rines, P. K. Sorger, and G. Danuser, "Automatic fluorescent tag detection in 3D with super-resolution: Application to the analysis of chromosome movement," J. Microscopy, vol. 208, no. 1, pp. 49-64, 2002.

[208] A. Raj, P. van den Bogaard, S. A. Rifkin, A. van Oudenaarden, and S. Tyagi, "Imaging individual mRNA molecules using singly labeled probes," Nat. Meth., vol. 10, no. 5, pp. 877-879, 2008.

[209] A. Basset, J. Boulanger, P. Bouthemy, C. Kervrann, and J. Salamero, "SLT-LoG: A vesicle segmentation method with automatic scale selection and local thresholding applied to tirf microscopy," in Proc. IEEE Int. Symp. Biomed. Imag. (ISBI), Beijing, China, 2014, pp. 533-536.

[210] H.-F. Yang, C.-S. Chen, and X. Descombes, "Detection and tracking of Golgi outposts in microscopy data," in Proc. IEEE Int. Symp. Biomed. Imag. (ISBI), New York, NY, USA, 2015, pp. 793-796.

[211] J.-C. Olivo-Marin, "Extraction of spots in biological images using multi-scale products," Pattern Recogn., vol. 35, no. 9, pp. 1989-1996, 2002.

[212] V. Racine, M. Saschse, J. Salamero, V. Fraisier, A. Trubuil, and J.-B. Sibarita, "Visualization and quantification of vesicle trafficking on a 3D cytoskeleton network in living cells," J. Microscopy, pp. 214-228, 2007.

[213] P. Ruusuvuori et al., "Evaluation of methods for detection of fluorescence labeled subcellular objects in microscope images," BMC Bioinformatics, vol. 11 , no. 248, 2010.

[214] S. G. Mallat, "A theory for multiresolution signal decomposition: The wavelet representation," IEEE Trans. Pattern Anal. Mach. Intell., vol. 11, no. 7, pp. 674-693, Jul. 1989.

[215] J. Serra, Image Analysis and Mathematical Morphology. London, U.K.: Academic, 1982, vol. 1.

[216] L. Vincent, "Morphological grayscale reconstruction in image analysis: Applications and efficient algorithms," IEEE Trans. Image Process., vol. 2, no. 2, pp. 176-201, Apr. 1993.

[217] F. Meyer, "Contrast feature extraction," in Quant. Anal. Microstructures Mat. Sci. Biol. Medicine. Stuttgart, Germany: Riederer-Verlag, 1978, pp. 374-380.

[218] E. J. Breen, G. H. Joss, and K. L. Williams, "Locating objects of interest within biological images: The top hat box filter," Comp. Ass. Microscopy, vol. 3, no. 2, pp. 97-102, 1991.

[219] S. R. Sternberg, "Biomedical image processing," IEEE Trans. Comput., vol. C-16, no. 1, pp. 22-34, 1983.

[220] S. H. Rezatofighi, R. Hartley, and W. E. Hughes, "A new approach for spot detection in total internal reflection fluorescence microscopy," in Proc. IEEE Int. Symp. Biomed. Imag. (ISBI), Barcelona, Spain, 2012, pp. $860-863$.

[221] T. Crivelli, P. Bouthemy, B. Cernuschi-Frias, and J.-F. Yao, "Simultaneous motion detection and background reconstruction with a conditional mixed-state Markov random field," Int. J. Comp. Vis., vol. 94, no. 3, pp. 295-316, 2011.

[222] A. Criminisi, G. Cross, A. Blake, and K. Kolmogorov, "Bilayer segmentation of live video," in Proc. IEEE Comp. Vis. Pattern Recogn. (CVPR), New York, NY, USA, 2006, vol. 1, pp. 53-60.

[223] V. Cevher, A. Sankaranarayanan, M. F. Duarte, D. Reddy, R. G. Baraniuk, and R. Chellappa, "Compressive sensing for background subtraction," in Proc. Eur. Conf. Comp. Vis. (ECCV), Marseille, France, 2008, vol. 2 , pp. $155-168$.

[224] E. C. Hall and R. M. Willett, "Foreground and background reconstruction in Poisson video," in Proc. IEEE Int. Conf. Image Process. (ICIP), Melbourne, Australia, 2013, pp. 2484-5488.

[225] J. Boulanger, C. Kervrann, and P. Bouthemy, "Estimation of dynamic background for fluorescence video-microscopy," in Proc. IEEE Int. Conf. Image Process. (ICIP), Atlanta, GA, USA, 2006, pp. 2509-2512.

[226] T. Pecot, P. Bouthemy, J. Boulanger, A. Chessel, S. Bardin, J. Salamero, and C. Kervrann, "Background fluorescence estimation and vesicle segmentation in live cell imaging with conditional random fields," IEEE Trans. Image Process., vol. 4, no. 2, pp. 667-680, Feb. 2015.
[227] P. Rosin, "Thresholding for change detection," Comp. Vis. Image Understand., vol. 86, pp. 79-95, 2002.

[228] A. Gidon et al., "Dynamics of Rab11a-MyoVb-FIP2 complex frames the late recycling steps of Langerin from the ERC to the plasma membrane," Traffic, vol. 13, pp. 815-833, 2012.

[229] E. M. Manders, F. J. Verbeek, and J. A. Aten, "Measurement of co-localization of objects in dual-color confocal images," J. Microsc., vol. 169, pp. 375-382, 1993.

[230] J. A. Helmuth, G. Paul, and I. F. Sbalzarini, "Beyond co-localization: Inferring spatial interactions between sub-cellular structures from microscopy images," BMC Bioinformatics, vol. 11, no. 1, p. 372, 2010.

[231] T. Lagache, G. Lang, N. Sauvonnet, and J.-C. Olivo-Marin, "Analysis of the spatial organization of molecules with robust statistics," PLoS One, vol. 8, no. 12, p. e80914, 2013.

[232] B. D. Ripley, "Modeling spatial patterns," J. Roy. Statist. Soc. Ser. B, vol. 39, no. 2, pp. 172-212, 1977.

[233] B. Zhang, N. Chenouard, J.-C. Olivo-Marin, and V. Meas-Yedid, "Statistical colocalization in biological imaging with false discovery control," in Proc. IEEE Int. Symp. Biomed. Imag. (ISBI), Paris, France, 2008, pp. 1327-1330.

[234] D. Nair, E. Hosy, J. D. Petersen, A. Constals, G. Giannone, D. Choquet, and J.-B. Sibarita, "Super-resolution imaging reveals that ampa receptors inside synapses are dynamically organized in nanodomains regulated by psd95," J. Neurosci., vol. 33, no. 32, pp. 13204-13224, 2013.

[235] I. Izeddin et al., "Wavelet analysis for single molecule localization microscopy," Opt. Expr., vol. 20, no. 3, pp. 2081-2095, 2012.

[236] H. Qian, M. P. Sheetz, and E. L. Elson, "Single particle tracking: Analysis of diffusion and flow in two-dimensional system," Biophys. J., vol. 60, pp. 910-921, 1991.

[237] T. Bickel, "A note on confined diffusion," Phys. A, Statist. Mech. App., vol. 377, no. 1, pp. 24-32, 2007.

[238] M. J. Saxton, "Single-particle tracking: Models of directed transport," Biophys. J., vol. 67, no. 5, pp. 2110-2119, 1994

[239] A. Caspi, R. Granek, and M. Elbaum, "Diffusion and directed motion in cellular transport," Phys. Rev. E, vol. 66, no. 1, p. 011916, 2002.

[240] M. J. Saxton, "Lateral diffusion in an archipelago. single-particle diffusion," Biophys. J., vol. 64, pp. 1766-1780, 1993.

[241] D. Nair, E. Hosy, J. D. Petersen, A. Constals, G. Giannone, D. Choquet, and J.-B. Sibarita, "Mean square displacement analysis of single-particle trajectories with localization error: Brownian motion in isotropic medium," Phys. Rev. E Stat. Nonlin. Soft. Matter Phys., vol. 82, no. 4, p. 041914,2010, Pt 1.

[242] B. Hebert, S. Costantino, and P. W. Wiseman, "Spatiotemporal image correlation spectroscopy (STICS) theory, verification, and application to protein velocity mapping in living cho cells," Biophys. J., vol. 88, no. 5, pp. 3601-3614, 2005 .

[243] L. Ji and G. Danuser, "Tracking quasi-stationary flow of weak fluorescent signals by adaptive multi-frame correlation," J. Microscopy, vol. 220, no. 3, pp. 150-167, 2005.

[244] D. L. Kolin and P. W. Wiseman, "Advances in image correlation spectroscopy: Measuring number densities, aggregation states, and dynamics of fluorescently labeled macromolecules in cells," Cell Biochem. Biophys., vol. 49, no. 3, pp. 141-164, 2007.

[245] N. Chenouard, I. Bloch, and J.-C. Olivo-Marin, "Multiple hypothesis tracking for cluttered biological image sequences," IEEE Trans. Pattern Anal. Mach. Intell., vol. 35, no. 11, pp. 2736-2750, Nov. 2013.

[246] I. Smal et al., "Multiple object tracking in molecular bioimaging by Rao-Blackwellized marginal particle filtering," Med. Image Anal., vol. 12 , no. 6 , pp. $764-777,2008$.

[247] I.-H. Kim, Y.-C. M. Chen, D. L. Spector, R. Eils, and K. Rohr, "Nonrigid registration of 2D and 3D dynamic cell nuclei images for improved classification of subcellular particle motion," IEEE Trans. Image Process., vol. 20, no. 4, pp. 1011-1022, Apr. 2011.

[248] J. Delpiano, J. Jara, J. Scheer, O. A. Ramírez, J. Ruiz-del Solar, and S. Härtel, "Performance of optical flow techniques for motion analysis of fluorescent point signals in confocal microscopy," Mach. Vis. Applicat., vol. 23, no. 4, pp. 675-689, 2011.

[249] J. Hubený, V. Ulman, and P. Matula, "Estimating large local motion in live-cell imaging using variational optical flow-towards motion tracking in live cell imaging using optical flow," in Proc. Int. Conf. Comput. Vis. Theory Applicat. (VISAPP), Barcelona, Spain, 2007, pp. 542-548.

[250] S. Baker, D. Scharstein, J. P. Lewis, S. Roth, M. J. Black, and R. Szeliski, "A database and evaluation methodology for optical flow," Int. J. Comp. Vis., pp. 1-31, 2011. 
[251] L. Pizarro, J. Delpiano, P. Aljabar, J. Ruiz del Solar, and D. Rueckert, "Towards dense motion estimation in light and electron microscopy," in Proc. IEEE Int. Symp. Biomed. Imag. (ISBI), Chicago, IL, USA, 2011, pp. 1939-1942.

[252] T. Lecomte, R. Thibeaux, N. Guillen, A. Dufour, and J.-C. OlivoMarin, "Fluid optical flow for forces and pressure field estimation in cellular biology," in Proc. IEEE Int. Conf. Image Process. (ICIP), Orlando, FL, USA, 2012, pp. 69-72.

[253] F. Amat, E. W. Myers, and P. J. Keller, "Fast and robust optical flow for time-lapse microscopy using super-voxels," Bioinformatics, vol. 29, no. 3, pp. 373-380, 2013.

[254] L. Guignard, C. Godin, U.-M. Fiuza, L. Hufnagel, P. Lemaire, and G. Malandain, "Spatio-temporal registration of embryo images," in Proc. IEEE Int. Symp. Biomed. Imag. (ISBI'14), Beijing, China, 2014, pp. $778-781$.

[255] S. Ozeré, P. Bouthemy, F. Spindler, P. Paul-Gilloteaux, and C. Kervrann, "Robust parametric stabilization of moving cells with intensity correction in light microscopy image sequences," in Proc. IEEE Int. Symp. Biomed. Imag. (ISBI), San Francisco, CA, USA, 2013, pp. 468-471.

[256] H. Zimmer, A. Bruhn, and J. Weickert, "Optic flow in harmony," Int. J. Comput. Vis., vol. 93, no. 3, pp. 368-388, 2011.

[257] D. Fortun, P. Bouthemy, P. Paul-Gilloteaux, and C. Kervrann, “Aggregation of patch-based estimations for illumination-invariant optical flow in live cell imaging," in Proc. IEEE Int. Symp. Biomed. Imag. (ISBI), San Francisco, CA, USA, 2013, pp. 660-663.

[258] D. Fortun, C. Chen, P. Paul-Gilloteaux, F. Waharte, J. Salamero, and C. Kervrann, "Correlation and variational approaches for motion and diffusion estimation in fluorescence imaging," in Proc. IEEE Eur. Signal Process. Conf. (EUSIPCO), Marrakech, Marroco, 2013, pp. 1-5.

[259] D. M. Soumpasis, "Theoretical analysis of fluorescence photobleaching recovery experiments," Biophys. J., vol. 41, no. 1, pp. 95-97, 1983.

[260] S. A. Kim, H. Sanabria, M. A. Digman, E. Gratton, P. Schwille, W. R. Zipfel, and M. N. Waxham, "Quantifying translational mobility in neurons: Comparison between current optical techniques," J. Neurosci., vol. 30, no. 49, pp. 16409-16416, 2010.

[261] R. Ananthakrishnan and A. Ehrlicher, "The forces behind cell movement," Int. J. Biol. Sci., vol. 3, no. 5, pp. 303-317, 2007.

[262] B. Maugis, J. Brugues, P. Nassoy, N. Guillen, P. Sens, and F. Amblard, "Dynamic instability of the intracellular pressure drives bleb-based motility," J. Cell Sci., no. 123, pp. 3884-3892, 2010.

[263] K. Khairy, J. Foo, and J. Howard, "Shapes of Red Blood Cells: Comparison of 3D Confocal Images with the Bilayer-couple Model," Cell. Mol. Bioeng., vol. 1, pp. 173-181, 2008.

[264] C. Ducroz, J.-C. Olivo-Marin, and A. Dufour, "Spherical Harmonics based extraction and annotation of cell shape in 3D time-lapse microscopy sequences," in Proc. IEEE Eng. Med. Biol. Soc. (EMBC), Boston, MA, USA, 2011, pp. 6619-6622.

[265] A. Dufour, R. Thibeaux, E. Labruyère, N. Guillén, and J.-C. OlivoMarin, "3D active meshes: Fast discrete deformable models for cell tracking in 3D time-lapse microscopy," IEEE Trans. Image Process., vol. 20, no. 7, pp. 1925-1937, Jul. 2011.

[266] M. Kazhdan, T. Funkhouser, and S. Rusinkiewicz, "Rotation invariant spherical harmonic representation of 3D shape descriptors," in Proc. Eur. Symp. Geometry Process. (SGP), Aachen, Germany, 2003, pp. $156-164$.

[267] A. A. Dima et al., "Comparison of segmentation algorithms for fluorescence microscopy images of cells," Cytometry A, vol. 79, no. 7, pp. 545-559, 2011.

[268] A. Dufour, V. Shinin, S. Tajbakhsh, N. Guillén-Aghion, J.-C. OlivoMarin, and C. Zimmer, "Segmenting and tracking fluorescent cells in dynamic 3-D microscopy with coupled active surfaces," IEEE Trans. Image Process., vol. 14, no. 9, pp. 1396-1410, Sep. 2005.

[269] O. Dzyubachyk, W. A. van Cappellen, J. Essers, W. J. Niessen, and E. Meijering, "Advanced level-set-based cell tracking in time-lapse fluorescence microscopy,", IEEE Trans. Med. Imag., vol. 29, no. 3, pp. 852-867, Mar. 2010.

[270] C. Zimmer, E. Labruyere, V. Meas-Yedid, N. Guillen, and J.-C. OlivoMarin, "Segmentation and tracking of migrating cells in videomicroscopy with parametric active contours: A Tool for cell-based drug testing," IEEE Trans. Med. Imag., vol. 21, no. 10, pp. 1212-1221, 2002.

[271] M. Kass, A. P. Witkin, and D. Terzopoulos, "Snakes: Active contour models," Int. J. Comput. Vis., vol. 1, no. 4, pp. 321-331, 1988.
[272] C. Zimmer and J.-C. Olivo-Marin, "Coupled parametric active contours," IEEE Trans. Pattern Anal. Mach. Intell., vol. 27, no. 11, pp. 1838-1842, Nov. 2005.

[273] R. Malladi, J. A. Sethian, and B. C. Vemuri, "Shape modeling with front propagation: A level set approach," IEEE Trans. Pattern Anal. Mach. Intell., vol. 17, no. 2, pp. 158-175, Feb. 1995.

[274] E. Meijering, O. Dzyubachyk, I. Smal, and W. A. van Cappellen, "Tracking in cell and developmental biology," Semin. Cell Dev. Biol., vol. 20, no. 8, pp. 894-902, 2009.

[275] D. Padfield, J. Rittscher, and B. Roysam, "Coupled minimum-cost flow cell tracking for high-throughput quantitative analysis," Med. Image Anal., vol. 15, no. 4, pp. 650-668, 2011.

[276] M. Maska, O. Danek, S. Garasa, A. Rouzaut, A. Munoz-Barrutia, and C. Ortiz-de-Solorzano, "Segmentation and shape tracking of whole fluorescent cells based on the Chan-Vese model," IEEE Trans. Med. Imag., vol. 32, no. 6, pp. 995-1006, Jun. 2013.

[277] C.-J. Du, J. G. Ferguson, P. T. Hawkins, L. R. Stephens, and T. Bretschneider, "Local shape representation in 3D: From weighted spherical harmonics to spherical wavelets," in Proc. British Mach. Vis. Conf. (BMVC), Surrey, U.K., 2012, pp. 1-12.

[278] K. Khairy, J. Foo, and J. Howard, "Shapes of red blood cells: Comparison of 3D confocal images with the bilayer-couple model," Cell. Mol. Bioeng., vol. 1, no. 2-3, pp. 173-181, 2010.

[279] C. Ducroz, J.-C. Olivo-Marin, and A. Dufour, "Characterization of cell shape and deformation in 3D using spherical harmonics," in Proc. IEEE Int. Symp. Biomed. Imag. (ISBI), Barcelona, Spain, 2012, pp. $848-851$.

[280] C.-J. Du, P. T. Hawkins, L. R. Stephens, and T. Bretschneider, “3D time series analysis of cell shape using Laplacian approaches," $B M C$ Bioinformatics, vol. 14, p. 296, 2013.

[281] A. Dufour, T.-Y. Liu, C. Ducroz, C. R. Tournemenne, B. Cummings, R. Thibeaux, N. Guillen, A. O. Hero, and J.-C. Olivo-Marin, "Signal processing challenges in quantitative 3-D cell morphology: More than meets the eye,," IEEE Signal Process. Mag., vol. 32, no. 1, pp. 30-40, Jan. 2015

[282] D. Gerlich, J. Mattes, and R. Eils, "Quantitative motion analysis and visualization of cellular structures," Method, vol. 29, no. 1, 2003.

[283] P. V. Belichenko, A. Oldfors, B. Hagberg, and A. Dahlström, "Rett syndrome: 3-D confocal microscopy of cortical pyramidal dendrites and afferents," Neuroreport, vol. 5, no. 12, pp. 1509-1513, 1994.

[284] N. Kasthuri and J. W. Lichtman, "The role of neuronal identity in synaptic competition," Nature, vol. 424, no. 6947, pp. 426-430, 2003.

[285] J. Chen and B. G. Condron, "Drosophila serotonergic varicosities are not distributed in a regular manner," J. Comp. Neurol., vol. 515, no. 4, pp. 441-453, 2009

[286] E. A. Daubert, D. S. Heffron, J. W. Mandell, and B. G. Condron, "Serotonergic dystrophy induced by excess serotonin," Mol. Cell. Neurosci., vol. 44, no. 3, pp. 297-306, 2010.

[287] H. Cuntz, F. Forstner, J. Haag, and A. Borst, "The morphological identity of insect dendrites," PLoS Comput. Biol., vol. 4, no. 12, p. e1000251, 2008.

[288] C. Koch and I. Segev, "The role of single neurons in information processing," Nat. Neurosci., vol. 3, pp. 1171-1177, 2000.

[289] J. Chen and B. G. Condron, "Branch architecture of the fly larval abdominal serotonergic neurons," Dev. Biol., vol. 320, no. 1, pp. 30-38, 2008.

[290] M. Oberlaender, R. M. Bruno, B. Sakmann, and P. J. Broser, "Transmitted light brightfield mosaic microscopy for three-dimensional tracing of single neuron morphology," J. Biomed. Opt., vol. 12, no. 6 , p. $64029,2007$.

[291] A. Santamaria-Pang, C. M. Colbert, P. Saggau, and I. A. Kakadiaris, "Automatic centerline extraction of irregular tubular structures using probability volumes from multiphoton imaging," in Proc. Med. Image Comput. Comput.-Assisted Intervent. (MICCAI), Brisbane, Australia, 2007, pp. 486-494.

[292] G. Knott, H. Marchman, D. Wall, and B. Lich, "Serial section scanning electron microscopy of adult brain tissue using focused ion beam milling," J. Neurosci., vol. 28, no. 12, pp. 2959-2964, 2008.

[293] K. A. Al-Kofahi, A. Can, S. Lasek, D. H. Szarowski, N. DowellMesfin, W. Shain, J. N. Turner, and B. Roysam, "Median-based robust algorithms for tracing neurons from noisy confocal microscope images," IEEE Trans. Inf. Technol. Biomed., vol. 7, no. 4, pp. 302-317, Dec. 2003.

[294] J. Xie, T. Zhao, T. Lee, E. Myers, and H. Peng, "Anisotropic path searching for automatic neuron reconstruction," Med. Image Anal., vol. 15 , no. 5, pp. 680-689, 2011. 
[295] K. A. Al-Kofahi, A. Can, S. Lasek, D. H. Szarowski, N. DowellMesfin, W. Shain, J. N. Turner, and B. Roysam, "Median-based robust algorithms for tracing neurons from noisy confocal microscope images," IEEE Trans. Inf. Technol. Biomed., vol. 7, no. 4, pp. 302-317, Dec. 2003

[296] G. González, E. Turetken, F. Fleuret, and P. Fua, "Delineating trees in noisy 2D images and 3D image-stacks," in Proc. Comp. Vis. Pattern Recogn. (CVPR), San Francisco, CA, USA, 2010, pp. 2799-2806.

[297] A. F. Frangi, W. J. Niessen, K. L. Vincken, and M. A. Viergever, "Multiscale vessel enhancement filtering," in Proc. Med. Image Comput. Comput.-Assisted Intervent. (MICCAI), Cambridge, MA, USA, 1998, pp. $130-137$.

[298] Y. Wang, A. Narayanaswamy, C.-L. Tsai, and B. Roysam, "A broadly applicable 3-D neuron tracing method based on open-curve snake," Neuroinformatics, vol. 9, no. 2-3, pp. 193-217, 2011.

[299] S. Mukherjee, B. Condron, and S. T. Acton, "Tubularity flow field: A technique for automatic neuron segmentation," IEEE Trans. Image Process., vol. 24, no. 1, pp. 374-389, 2015.

[300] S. Basu, A. Aksel, B. Condron, and S. T. Acton, "Segmentation and tracing of singlar neurons from $3 \mathrm{D}$ confocal microscope images," IEEE J. Biomed. Heath Informatics, vol. 17, no. 2, pp. 319-335, 2013.

[301] G. González, F. Aguet, F. Fleuret, M. Unser, and P. Fua, "Steerable features for statistical 3D dendrite detection," in Proc. Med. Image Comput. Comput.-Assisted Intervent. (MICCAI), London, U.K., 2009, pp. 625-632.

[302] A. Dima, M. Scholz, and K. Obermayer, "Automatic segmentation and skeletonization of neurons from confocal microscopy images based on the 3-D wavelet transform," IEEE Trans. Image Process., vol. 11, no. 7, pp. 790-801, Nov. 2002.

[303] A. Mottini, X. Descombes, and F. Besse, "From curves to trees: A tree-like shapes distance using the elastic shape analysis framework," Neuroinformatics, vol. 13, no. 2, pp. 175-192, 2014.

[304] V. Jain, H. S. Seung, and S. C. Turaga, "Machines that learn to segment images: a crucial technology for connectomics," Curr. Opin. Neurobiol., vol. 20, no. 5, pp. 653-666, 2010.

[305] H. S. Seung and U. Sümbül, "Neuronal cell types and connectivity: Lessons from the retina," Neuron, vol. 83, no. 6, pp. 1262-1272, 2014.

[306] E. Serradell, P. Glowacki, J. Kybic, F. Moreno-Noguer, and P. Fua, "Robust non-rigid registration of 2D and 3D graphs," in Proc. IEEE Conf. Comput. Vis. Pattern Recogn. (CVPR), Providence, RI, USA, 2012, pp. 996-1003, ISA.

[307] E. Serradell, M. A. Pinheiro, R. Sznitman, J. Kybic, F. Moreno-Noguer, and P. Fua, "Non-rigid graph registration using active testing search," IEEE Trans. Pattern Anal. Mach. Intell., vol. 37, no. 3, pp. 625-638, Mar. 2015.

[308] S. Basu, B. Condron, and S. T. Acton, "Path2Path: Hierarchical PathBased analysis for neuron matching," in Proc. IEEE Int. Symp. Biomed. Imag. (ISBI), Chicago, IL, USA, 2011, pp. 996-999.

[309] U. Sümbül, A. Zlateski, A. Vishwanathan, R. H. Masland, and H. S. Seung, "Automated computation of arbor densities: A step toward identifying neuronal cell types," Front. Neuroanat., vol. 8, 2014.

[310] S. Mukherjee, S. Basu, B. Condron, and S. T. Acton, "A geometricstatistical approach toward neuron matching," in Proc. IEEE Int. Symp. Biomed. Imag. (ISBI), Barcelona, Spain, 2012, pp. 772-775.

[311] X. Xu and S. T. C. Wong, "Optical microscopic image processing of dendritic spines morphology,” IEEE Signal Process. Mag., vol. 23, no. 4, pp. 132-135, Jul. 2006.

[312] A. Rodriguez, D. B. Ehlenberger, D. L. Dickstein, P. R. Hof, and S. L. Wearne, "Automated three-dimensional detection and shape classification of dendritic spines from fluorescence microscopy images," PLoS One, vol. 3, no. 4, p. e1997, 2008.

[313] Q. Li and Z. Deng, "A surface-based 3-D dendritic spine detection approach from confocal microscopy images," IEEE Trans. Image Process., vol. 21, no. 3, pp. 1223-1230, Mar. 2012.

[314] T. He, Z. Xue, Y. Kim, and S. T. Wong, "Three-dimensional dendritic spine detection based on minimal cross-sectional curvature," in Proc. Int. Symp. Biomed. Imag. (ISBI), Barcelona, Spain, 2012, pp. 1639-1642.

[315] L. Rada, E. Erdil, A. O. Argunsah, D. Unay, and M. Cetin, "Automatic dendritic spine detection using multiscale dot enhancement filters and SIFT features," in Proc. IEEE Int. Conf. Image Process. (ICIP), Paris, France, 2014, pp. 26-30.

[316] P. Shi, X. Zhou, Q. Li, M. Baron, M. A. Teylan, Y. Kim, and S. T. C. Wong, "Online three-dimensional dendritic spines mophological classification based on semi-supervised learning," in Proc. IEEE Int. Symp. Biomed. Imag. (ISBI), Boston, MA, USA, 2009, pp. 1019-1022.
[317] Q. Li, Z. Deng, Y. Zhang, X. Zhou, U. V. Nagerl, and S. T. C. Wong, “A global spatial similarity optimization scheme to track large numbers of dendritic spines in time-lapse confocal microscopy," IEEE Trans. Med. Imag., vol. 30, no. 3, pp. 632-641, Mar. 2011.

[318] A. Kreshuk, C. N. Straehle, C. Sommer, U. Koethe, M. Cantoni, G. Knott, and F. A. Hamprecht, "Automated detection and segmentation of synaptic contacts in nearly isotropic serial electron microscopy images," PLoS One, vol. 6, no. 10, p. e24899, 2011.

[319] I. Carlbom, D. Terzopoulos, and K. M. Harris, "Computer-assisted registration, segmentation, and 3D reconstruction from images of neuronal tissue sections," IEEE Trans. Med. Imag., vol. 13, no. 2, pp. 351-362, 1994.

[320] H. Nguyen and Q. Ji, "Shape-driven three-dimensional watersnake segmentation of biological membranes in electron tomography," IEEE Trans. Med. Imag., vol. 27, no. 5, pp. 616-628, May 2008.

[321] C. Becker, K. Ali, G. Knott, and P. Fua, "Learning context cues for synapse segmentation," IEEE Trans. Med. Imag., vol. 32, no. 10, pp. 1864-1877, Oct. 2013.

[322] C. Becker, C. Christoudias, and P. Fua, "Domain adaptation for microscopy imaging," IEEE Trans. Med. Imag., vol. 34, no. 5, pp. 1125-1139, May 2014.

[323] O. Ghita, J. Dietlmeier, and P. F. Whelan, "Automatic segmentation of mitochondria in EM data using pairwise affinity factorization and graph-based contour searching," IEEE Trans. Image Process., vol. 23, no. 10 , pp. $4576-4586$, Oct. 2014.

[324] A. Jorstad and P. Fua, "Refining mitochondria segmentation in electron microscopy imagery with active surfaces," in Proc. ECCV Workshop Non-Rigid Shape Anal. Deformable Image Alignment, 2014, no. EPFLCONF-201868.

[325] A. Lucchi, K. Smith, R. Achanta, G. Knott, and P. Fua, "Supervoxelbased segmentation of mitochondria in EM image stacks with learned shape features," IEEE Trans. Med. Imag., vol. 31, no. 2, pp. 474-486, Feb. 2012.

[326] G. A. Ascoli, D. E. Donohue, and M. Halavi, "NeuroMorpho. Org: A central resource for neuronal morphologies," J. Neurosci., vol. 27, no. 35, pp. 9247-9251, 2007.

[327] K. M. Brown et al., "The DIADEM data sets: representative light microscopy images of neuronal morphology to advance automation of digital reconstructions," Neuroinformatics, vol. 9, no. 2-3, pp. 143-157, 2011.

[328] H. Shen, "Neuron encyclopaedia fires up to reveal brain secrets," $\mathrm{Na}$ ture, vol. 520, pp. 13-14, 2015.

[329] C. Lavelle et al., "From molecules to organisms: Towards multiscale integrated model of biological systems," Theoret. Biol. Insights, vol. 1, pp. 13-22, 2008.

[330] J. R. Swedlow, I. Goldberg, E. Brauner, and P. K. Sorger, "Image informatics and quantitative analysis in biological imaging," Science, vol. 300, no. 5616, pp. 100-102, 2003.

[331] V. Wiesmann, D. Franz, C. Held, C. Münzenmayer, R. Palmisano, and T. Wittenberg, "Review of free software tools for image analysis of fluorescence cell micrographs," J. Microscopy, vol. 257, no. 1, pp. 39-53, 2014.

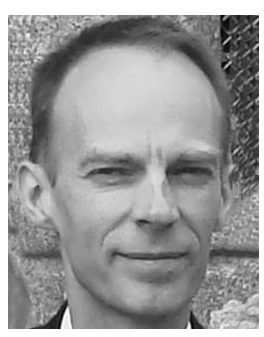

Charles Kervrann received the M.Sc. (1992), the Ph.D. (1995), and the HDR (Habilitation à Diriger des Recherches, 2010) in signal processing and telecommunications from the University of Rennes 1, France. From 1997 to 2010, he was Researcher at the INRA Applied Mathematics and Informatics Department (1997-2003, Jouy-en-Josas, France) and he joined the VISTA Inria research group in 2003 (Rennes, France). In 2010, he was appointed to the rank of Research Director, Inria Research Centre in Rennes. His is currently the head of the Serpico (Space-timE RePresentation, Imaging and cellular dynamics of molecular COmplexes) research group. His current research interests include mathematical and statistical methods for biological image processing and microscopy. His work focuses on image sequence analysis, motion estimation, object detection, noise modeling for microscopy and traffic, and dynamics modeling in cell biology. He has served as member of the program committees of the major conferences in image processing and computer vision. He is member of the editorial board of IEEE SIGNAL PROCESSING LETTERS, member of the IEEE BISP (Bio Imaging and Signal Processing) technical committee and co-head of the IPDM-BioImage Informatics node of the french national infrastructure France-BioImaging. 


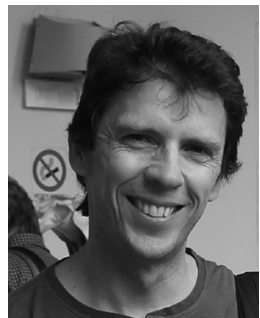

Carlos Óscar Sánchez Sorzano received the B.Sc. and M.Sc. electrical engineering with two specialities (electronics and networking, University of Málaga), the B.Sc. in computer science (University of Málaga), the B.Sc. and M.Sc. in mathematics, (speciality in statistics, UNED), the $\mathrm{Ph} . \mathrm{D}$. in biomedical engineering (University of Politácnica de Madrid), and the Ph.D. in pharmacy (University of San Pablo-CEU). He served as secretary of the Department of Engineering of Electronic and Telecommunication Systems of the University CEU-San Pablo (Madrid) between 2005 and 2008, Coordinator of the Section on Signal and Communications theory between 2004 and 2009, Head of the Bioengineering Laboratory of that University between 2007 and 2008, Director of the Summerschool on Advanced Data Analysis and Modelling between 2006 and 2009, and codirector of the Master on Computational Biotechnology between 2007 and 2009. He did his Ph.D. at the Biocomputing Unit of the National Center of Biotechnology (CSIC), and a Post-Doc at the Biomedical Imaging Group of the Swiss Federal Institute of Technology Lausanne (EPFL). In 2006 he received the Ángel Herrera research prize. He has been a senior member of the IEEE since 2008 and that same year he was accredited as "profesor titular de universidad" by ANECA. In 2009 he was appointed as "Profesor Agregado" at University San Pablo CEU, awarded a Ramán y Cajal research contract and appointed as technical director of the INSTRUCT Image Processing Center for Microscopy. In 2011 and 2012 he was president of the National Association of Ramán y Cajal researchers. He coordinates the service of image processing and statistical analysis of the CNB since 2011. In 2013, he was accredited as Full Professor.

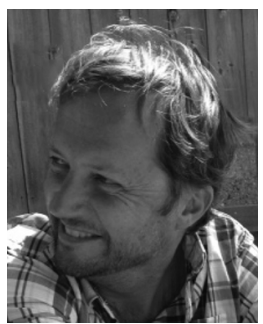

Scott T. Acton (F'13) was born in California. He is a Professor of Electrical and Computer Engineering and of Biomedical Engineering at the University of Virginia. He received his M.S. and Ph.D. degrees at the University of Texas at Austin. He received his B.S. degree at Virginia Tech. He is a Fellow of the IEEE "for contributions to biomedical image analysis."

Professor Acton's laboratory at UVA is called VIVA-Virginia Image and Video Analysis. They specialize in biological image analysis problems. The research emphases of VIVA include tracking, segmentation, representation, retrieval, classification, enhancement and image analysis problems in neuroscience. Recent theoretical interests include active contours, level sets, partial differential equation methods, scale space methods, graph signal processing and dictionary learning. Professor Acton has over 250 publications in the image analysis area including the books Biomedical Image Analysis: Tracking an Biomedical Image Analysis: Segmentation. Professor Acton currently serves as Editor-in-Chief of the IEEE TRANSACTIONS ON IMAGE PROCESSING.

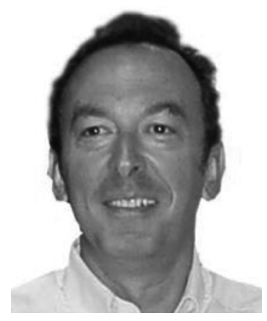

Jean-Christophe Olivo-Marin received the Ph.D and Habilitation à Diriger des Recherches degrees in optics and signal processing from the Institut d'Optique Théorique et Appliquée, University of ParisOrsay, France. He is the Head of the Bioimage Analysis Unit and the Director of the Center for Innovation and Technological Research at Institut Pasteur Paris. He has chaired the Cell Biology and Infection Department and was one of the cofounders of the Institut Pasteur Korea, Seoul, South Korea. Previous to that, he was a Staff Scientist at EMBL (Heidelberg). His research over the years has been centred about bioimage analysis, more specifically algorithms for multi-particle and cell tracking, microscope modelling, image deconvolution, and mathematical imaging. He is a strong proponent of reproducible research, which is at the heart of the Icy software (icy.bioimageanalysis.org). He is co-head of the IPDM-BioImage Informatics node of the french national infrastructure France-BioImaging. He is a Fellow of the IEEE, an IEEE SPS Distinguished Lecturer, chair of the IEEE International Symposium on Biomedical Imaging Steering Committee, and a member of the editorial boards of IEEE SIGNAL PROCESSING LETTERS, Medical Image Analysis and BMC Bioinformatics. He was the General Chair of the 2008 IEEE International Symposium on Biomedical Imaging.

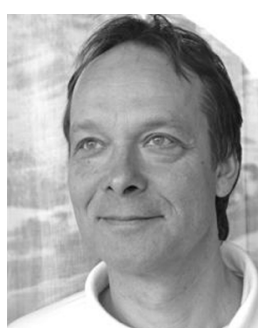

Michael Unser (M'89-SM'94-F'99) is Professor and Director of EPFL's Biomedical Imaging Group, Lausanne, Switzerland. His primary area of investigation is biomedical image processing. He is internationally recognized for his research contributions to sampling theory, wavelets, the use of splines for image processing, stochastic processes, and computational bioimaging. He has published over 250 journal papers on those topics. He is the author with P. Tafti of "An introduction to sparse stochastic processes", Cambridge University Press 2014.

From 1985 to 1997, he was with the Biomedical Engineering and Instrumentation Program, National Institutes of Health, Bethesda USA, conducting research on bioimaging.

Dr. Unser has held the position of associate Editor-in-Chief (2003-2005) for the IEEE TRANSACTIONS ON MEDICAL IMAGING. He is currently member of the editorial boards of SIAM Journal of Imaging Sciences, IEEE JOURNAL of Selected Topics IN Signal Processing, and Foundations and Trends in Signal Processing. He co-organized the first IEEE International Symposium on Biomedical Imaging (ISBI'2002) and was the founding chair of the technical committee of the IEEE-SP Society on Bio Imaging and Signal Processing (BISP).

Prof. Unser is a fellow of the IEEE (1999), an EURASIP fellow (2009), and a member of the Swiss Academy of Engineering Sciences. He is the recipient of several international prizes including three IEEE-SPS Best Paper Awards and two Technical Achievement Awards from the IEEE (2008 SPS and EMBS 2010). 